\title{
CHARACTERIZING DROPLET BREAKUP RATES OF SHEAR-THINNING DISPERSED PHASE IN MICROREACTORS
}

\author{
Voon-Loong Wong ${ }^{\mathrm{a}, \mathrm{b}, *, 1}$, Katerina Loizou ${ }^{\mathrm{b}, 2}$, Phei-Li Lau ${ }^{\mathrm{a}}$, Richard S. Graham ${ }^{\mathrm{c}}$, and \\ Buddhika N. Hewakandamby ${ }^{\mathrm{C}}$
}

${ }^{a}$ Department of Chemical and Environmental Engineering, University of Nottingham Malaysia Campus, Semenyih 43500, Malaysia

${ }^{\mathrm{b} D e p a r t m e n t}$ of Chemical and Environmental Engineering, University of Nottingham, Nottingham NG7 2RD, United Kingdom

'School of Mathematical Sciences, University of Nottingham, Nottingham NG7 2RD, United Kingdom

\section{ABSTRACT}

A two-phase flow predictive model with the integration of conservative level-set method (LSM) and Carreau-Yasuda constitutive equation was developed herein. The LSM was chosen as a potential interface capturing scheme for elucidating the interfacial phenomena including insight into the mechanism of shear-thinning droplets. In present paper, the dynamics of shear-dependent droplet emergence, growth, detachment and translocation in a Newtonian microsystem were examined via computational fluid dynamics (CFD) analysis. Dilute sodium carboxymethylcellulose ( $\mathrm{Na}-\mathrm{CMC})$ solution was treated as dispersed phase (70 $\mathrm{mPa} . \mathrm{s}<\eta_{o}<10.2644$ Pa.s) whereas the olive oil (68 mPa.s) was designated as continuous phase. Visualisation experiments were carried out and these laboratory data were used to validate the simulation results. Detailed $2 \mathrm{D}$ simulations were presented to examine systematically the impact of fluid properties on the droplet breakup rate at predefined flow rate ratio, $Q$ of 0.05 . The results yielded an inflection point in the dependence of droplet breakup rate on Na-CMC concentration was found in between the dilute and semi-dilute concentration regimes. This inflection point displays a non-monotonic profile which is mainly caused by the considerable viscosity effect of $\mathrm{Na}-\mathrm{CMC}$ polymer when its concentration increases above a critical

\footnotetext{
* Corresponding author; v.wong@hw.ac.uk (Voon-Loong Wong)

${ }^{1}$ Current address: School of Engineering and Physical Sciences, Heriot-Watt University Malaysia Campus, 62200 Putrajaya Wilayah Persekutuan, Malaysia.

${ }^{2}$ Current address: Department of Chemical Engineering, University College London, London, United Kingdom.
} 
value $\left(C>C^{*} \sim 0.40 \mathrm{wt} \%\right.$ ). This striking behaviour highlights the importance of rheological effects in flows with a shear-dependent fluid under various flow conditions. The viscous effect of $\mathrm{Na}-\mathrm{CMC}$ fluids substantially affects the manipulation over the droplet pinch-off time and production rate. Thus, it necessitate the control of the shear rate by adjusting the flow conditions and aspect ratio of microchannels.

Keywords: non-Newtonian; microfluidics; level-set; droplet breakup; T-junction geometry.

\section{Introduction}

Two-phase flow is a term covering the motion of two different interacting fluids that are in different phases such as liquid-liquid and liquid-vapor. In two-phase flow microfluidics, an emulsion contains a mixture of two immiscible liquids as one phase being dispersed throughout the other phase in small droplets. Most common emulsions include direct emulsions, oil droplets in an immiscible and continuous water phase, or inverted emulsions, water droplets in an immiscible and continuous oil phase. Emulsions are typically made by fissioning droplets with shear or impact and the resulting suspensions possess a wide size distribution of drop sizes (Umbanhowar, Prasad, \& Weitz, 2000). At low enough Reynolds (Re) number, a laminar flow regime is assumed and these droplets are translocate through microfluidic structures having dimensions most easily measured in microns. The manipulation of droplets in a confined microfluidic system has been highlighted as one of the earliest tools used in the fields of biomedical sciences. The characteristics of droplets become reliable tool for performing biological operations such as analyte encapsulation, sampling, metering, dilution, reaction and detection (Huebner et al., 2008; Niu \& deMello, 2012; Tawfik \& Griffiths, 1998; Theberge Ashleigh et al., 2010).

Droplets can be generated via a number of methods in microfluidic devices, including breakup in co-flowing stream(Cramer, Fischer, \& Windhab, 2004; Moon, Cheong, \& Choi, 2014; Utada, Fernandez-Nieves, Stone, \& Weitz, 2007), breakup in crossflowing stream (Garstecki, Fuerstman, Stone, \& Whitesides, 2006; Qiu, Silva, Tonkovich, \& Arora, 2010; Xu, Li, Tan, \& Luo, 2008), hydrodynamics flow-focusing (Anna \& Mayer, 2006; Peng, Yang, Guo, Liu, \& Zhao, 2011), and microchannel 
emulsification (Kobayashi, Nakajima, \& Mukataka, 2003; van der Zwan, Schroën, \& Boom, 2009; Yobas, Martens, Ong, \& Ranganathan, 2006). Cross-flowing in a Tjunction is one of the easiest microfluidic methods of generating highly monodispersed droplets. The formation of droplet at a T-junction, at which viscous shear-stresses induced by continuous stream of the horizontal channel overcome surface tension at the liquid-liquid interface and pull off droplets of the dispersed phase from the vertical channel. This is mainly due to the instabilities of free surface between the phases are sufficiently large. Thus, the size and frequency of the droplets can be accurately manipulated by modifying the relative pressures of the two immiscible liquid in order to enable the production of a wide range of vesicle shapes and patterns (Thorsen, Roberts, Arnold, \& Quake, 2001). Besides, opposed flowing (Shui, van den Berg, \& Eijkel, 2009) and perpendicular flowing (Leshansky \& Pismen, 2009) are another operation modes producing monodispersed droplet formation in a T-junction microchannel.

A numerical modelling approach to the multiphase flow problem provides a detailed and comprehensive description of the formation of microdroplets since a number of statistical information can be extracted from a predictive model. As the dimension of the interest gets smaller, the surface-based interfacial tension and the viscosity become more significant in controlling critical flow behavior of multiphase flow in microscale, especially when handling fluids that have a complex microstructure leading to non-Newtonian phenomena. Additionally, the non-Newtonian flow curve presents a nonlinear relationship between shear-stress and the rate of deformation. For instance, previous research efforts have been much devoted to the experimental analysis of the dynamics and relevant hydrodynamics of viscoelastic droplets (Arratia, Cramer, Gollub, \& Durian, 2009; Husny \& Cooper-White, 2006; Steinhaus, Shen, \& Sureshkumar, 2007) and few studies have focused on other sheardependent fluids such as purely viscous fluids and time-dependent fluids (Chhabra \& Richardson, 2008). Hitherto, there have been far fewer attempts to develop a predictive numerical model for the relevant physics of non-Newtonian droplets generation in a Newtonian bulk phases. However, there is no unique constitutive model that can represent the different characteristic behaviours of non-Newtonian fluids. 
In microfluidics, the geometry of droplet interface is usually complex and it can undergo large deformations or even topology changes such as fission and fusion in microchannel. There are two approaches, namely interface tracking (Hou, Lowengrub, \& Shelley, 2001; Tryggvason et al., 2001) and interface capturing (Bonometti \& Magnaudet, 2007), to represent the flow problem of droplet interface evolution or moving boundaries either explicitly or implicitly to the incompressible Navier-Stokes equation discretised on a fixed grid. Interface tracking of the moving boundary in multiphase system is an explicit representative that requires the computational meshes to track the evolving interface for each time-step. In contrast, the interface capturing approaches is an implicit representative that uses a phase function discretised on the fixed grid to represent the interface (Bonometti \& Magnaudet, 2007). In present paper, conservative level-set method (LSM) is adopted as it is a simple and robust scheme of interface capturing approaches for tracking moving interfaces and shapes (Osher \& Sethian, 1988). It permits numerical computations of such objects involving curves and surfaces to be performed on a fixed Cartesian grid without having to parameterize them (Olsson \& Kreiss, 2005; Olsson, Kreiss, \& Zahedi, 2007; Osher \& Sethian, 1988). In the LSM, the surface tension force is conventionally modelled as a distributed body force though concentrated in a band around the interfaces. The variation of surface tension force across the interface can be difficulties in the application of others common interface capturing methods, including the volume-of-fluid (Rider \& Kothe, 1998) (VOF) and lattice-Boltzmann method (LBM) (Takada, Misawa, Tomiyama, \& Fujiwara, 2000). However, the LSM can resolve to the challenges of mass conservation and the treatment of discontinuities across the flexible interface (Olsson \& Kreiss, 2005; Olsson et al., 2007).

The present paper demonstrates systematic sets of numerical simulations for the microdroplet generation of a shear-thinning $\mathrm{Na}-\mathrm{CMC}$ droplets in Newtonian flow at a microfluidic $T$ junction using a developed predictive computational model. The present model is adopted with the integration of conservative level-set approach and non-Newtonian constitutive law. Fundamental principles and application of microfluidic systems were presented due to the selection and interpretation of the subsequent numerical analysis. Numerical simulations of the Na-CMC microdroplets formation in an olive oil-based continuous phase were carried out. The present study 
reveals the interesting phenomena of shear-thinnings droplet formation during the systematic variation in flow rates, interfacial tension, and surface wettability. As a result, the rheological characteristics of $\mathrm{Na}-\mathrm{CMC}$ solution are strongly depending on its concentration. Thus, the impact of these rheological characteristics of $\mathrm{Na}-\mathrm{CMC}$ can be of great interest to provide an insightful understanding to the relevant physics of non-Newtonian droplet formation process in microfluidic flow.

\section{Experimental Methodology}

\subsection{Microfluidics Device Fabrication}

A channel dimension of $220 \mu \mathrm{m}\left(w_{c}\right) \times 90 \mu \mathrm{m}\left(w_{d}\right) \times 76 \mu \mathrm{m}(h)$ was used in present validation studies. It was fabricated in-house by photolithography and soft lithography technique. Fig. 1 illustrates the dimensions of a T-junction employed in the numerical and experimental studies. Prior to the start of fabrication process, an out sourced positive photolithographic mask (clear lines with a black background) was used to transfer of the photo-lithographically pattern onto the negative mould. The photolithographic mask with the desired layout of microchannel structure was designed using standard computer assisted design (CAD) program. The polydimethylsiloxane (PDMS) mould was fabricated by moulding a mixture of PDMS liquid pre-polymer, a 10:1 mixture of Sylgard 184 silicone elastomer and curing agent (Dow Corning, USA), onto the SU-8 master mould with SU-8 2025 (MicroChem Corporation, Newton, MA) as the photoresist that ultimately becomes the pattern on the silicon wafer. The glass slide with a cured PDMS thin layer and the surface of the PDMS mould with the micropattern indent then brought into conformal contact before flow experiment was conducted.

(b)

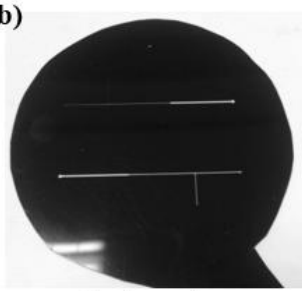

(c)

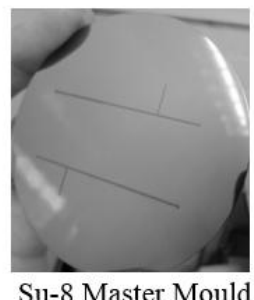

Su-8 Master Mould

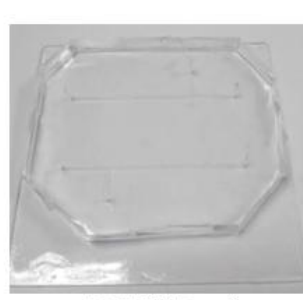

PDMS Device
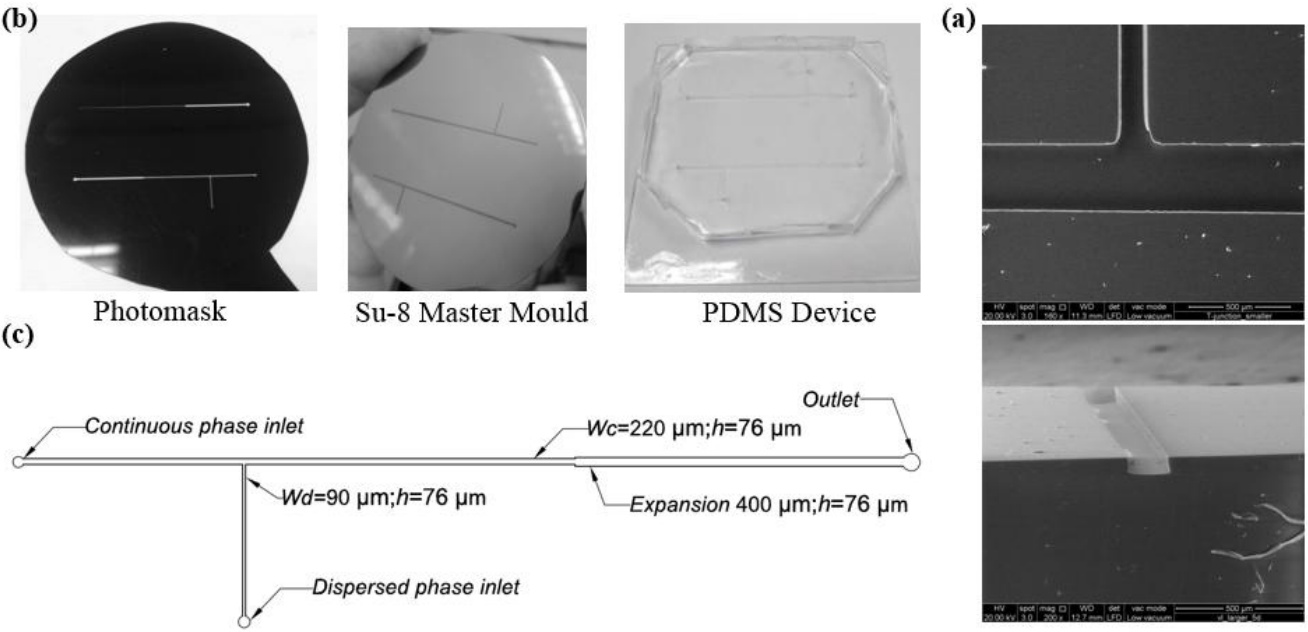

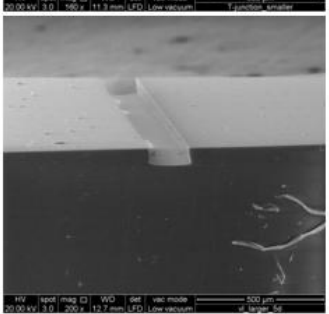


Fig. 1: Illustration of (a) microfluidics T-junction composed of rectangular channels; (b) schematic diagram of microfluidics T-junction composed of rectangular channel; (c) Scanning electron microscope (SEM) image of fabricated T-junction of PDMS microchannel and cross section of PDMS microchannel.

\subsection{Fluid Characterisation}

A calibrated BS/U-tube viscometer was used to perform the kinematic viscosity measurement for the transparent Newtonian olive oil (Sigma Aldrich). The capillary diameter of $B S / U$-tube viscometer with $0.50 \mathrm{~mm} \pm 0.01 \mathrm{~mm}$ was used. The viscometers were mounted upright in a beaker $(2000 \mathrm{~mL})$ of water at controlled room temperature $\left(20 \pm 2^{\circ} \mathrm{C}\right)$. Each sample solutions were allowed to attain the room temperature for 10 minutes. The viscosity measurements for Newtonian solution were conducted three times, and average values were taken for analysis. For shearthinnings $\mathrm{Na}-\mathrm{CMC}\left(\left[\mathrm{C}_{6} \mathrm{H}_{7} \mathrm{O}_{2}(\mathrm{OH}) \mathrm{CH}_{2} \mathrm{COONa}\right]_{\mathrm{n}}\right.$, Sigma Aldrich) aqueous solution, the rheological measurements were performed on controlled stress rheometer (MCR 302, Anton Paar) equipped with a cone-and-plate geometry (cone plate with diameter of $50 \mathrm{~mm}$; angle 0.04 radian) at controlled temperature of $20^{\circ} \mathrm{C}$. The samples were carefully loaded onto the measuring plate of the rheometer and left to idle for 10 minutes prior to viscosity measurement. Fig. 2 illustrates the shear viscosity against shear rate plot of $\mathrm{Na}-\mathrm{CMC}$ solutions at various concentrations ranging from $0.02 \mathrm{wt} \%$ to $1.20 \mathrm{wt} \%$ has been plotted over a log-log scale that covers nearly six orders of magnitude of shear rate (Wong, Loizou, Lau, Graham, \& Hewakandamby, 2017). In present work, power-law model is not potentially selected to describe the behaviour of shear-thinnings working fluid of $\mathrm{Na}-\mathrm{CMC}$ aqueous solution as it poses limitations on its range of applicability over a wide range of shear rate. In order to circumvent the drawback of the power-law model, alternative approaches such as Carreau-Yasuda model utilize viscosity functions that have finite values both at very low and high shear rate (Chhabra \& Richardson, 2008). Additionally, all the measurement data were well-fitted to the well-known CarreauYasuda model (Chhabra \& Richardson, 2008) for the shear-thinnings behaviour of Na-CMC:

$\eta(\dot{\gamma})=\eta_{\infty}+\left(\eta_{o}-\eta_{\infty}\right)\left[1+\left(\lambda_{C Y} \dot{\gamma}\right)^{a}\right]^{\frac{n-1}{a}}$ 
where $\eta_{0}$ is zero shear viscosity, $\eta_{\infty}$ is infinite shear viscosity, $\lambda_{C Y}$ is the relaxation time, $\dot{\gamma}$ is shear rate, $n$ is power-law exponent and $a$ is the fitting parameter for Carreau-Yasuda model.

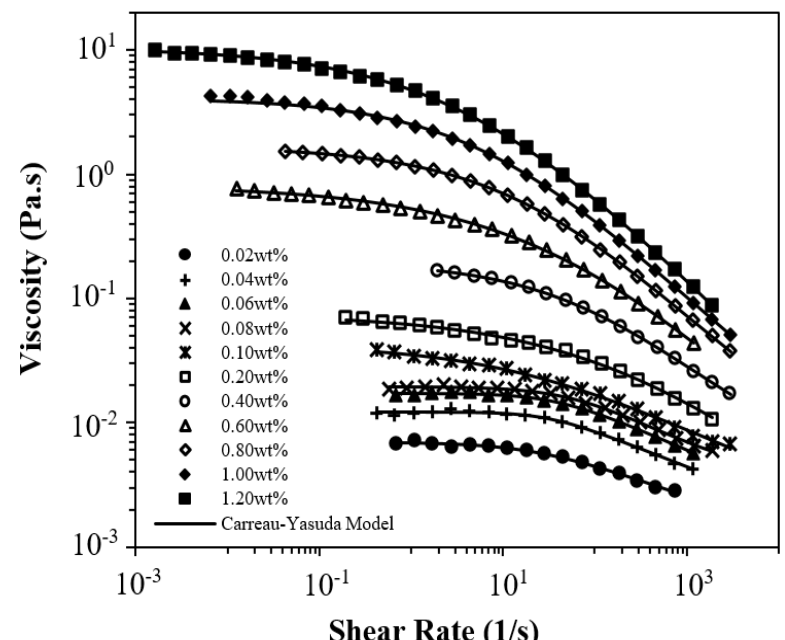

Fig. 2: Shear viscosity plotted against shear rate for a series of Na-CMC shearthinning solutions with different concentrations (Wong et al., 2017).

\subsection{Emulsification Setup}

The continuous (olive oil) and dispersed phase liquids (Na-CMC aqueous) were dispensed separately into the reservoirs of microchannel; each fluid was driven through microchannel at the desired continuous $\left(Q_{c}\right)$ and dispersed flow rates $\left(Q_{\mathrm{d}}\right)$ using syringe pumps (AL-1000, Florida and NE-1000, Netherlands), respectively. The processes before and after droplet formation in microfluidics device were recorded using a high speed camera (MIC Hotshot $1280 \mathrm{cc}$ ) connected to an epifluorescence microscope (Olympus IX51, Japan). The experimental setup with flow visualisation is illustrated in Fig. 3. After stabilizing the system for predetermined time intervals (20 minutes), videos were recorded at 500 frames per second (fps) after each flow rates of either continuous or dispersed phase were adjusted. The average effective droplet diameter of 30 droplets under experimental condition was measured through an image processing routine using MATLAB. All the collected data will be validated prior to the parametric analysis. 


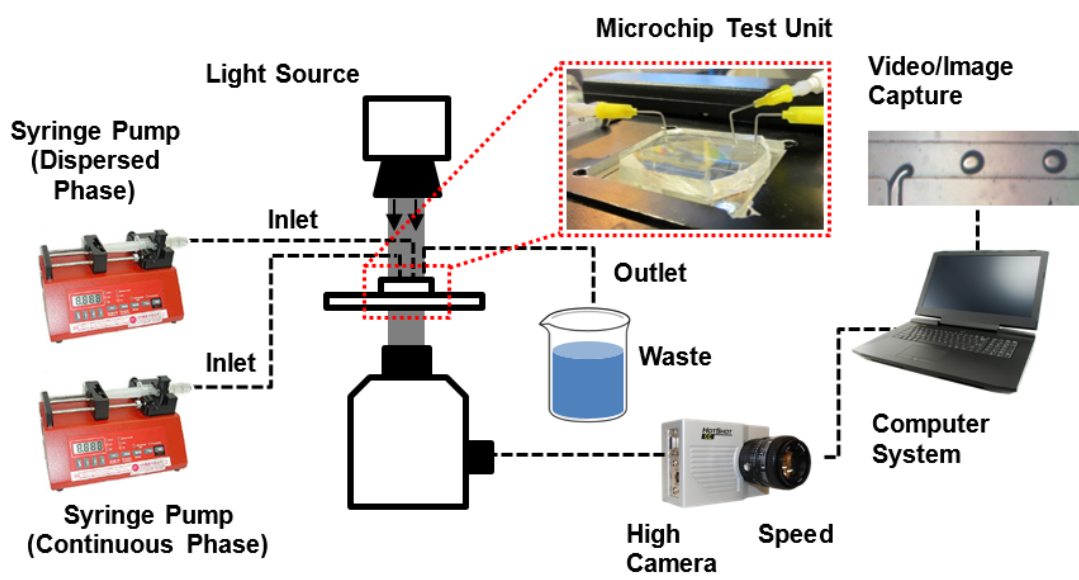

Fig. 3: Schematic diagram of experimental setup for flow visualization.

\section{CFD Modelling and Simulation}

\subsection{Theory Model}

A predictive numerical model was developed to track the fluid-fluid interfaces between two immiscible fluid phases of different density and viscosity. This applied the conservative LSM from CFD module using COMSOL Multiphysics. The mathematical model used in the computational fluidic dynamic simulation utilized a numerical time-stepping procedure to obtain the model behaviour over time. The governing equations for momentum and conservation laws of mass was considered, which was shown in the following forms with the assumption that the fluid is incompressible:

$\rho \frac{\partial \mathbf{u}}{\partial t}+\rho(\mathbf{u} \cdot \nabla) \mathbf{u}=-\nabla p+\nabla \cdot \eta\left(\nabla \mathbf{u}+(\nabla \mathbf{u})^{T}+F_{s t}\right.$

$\nabla \cdot \mathbf{u}=0$

where $\rho, \eta$, and $F_{s t}$ denote the density, dynamic viscosity, and the surface tension force respectively, $p$ denotes pressure while $I$ is the identity matrix. Naturally, the Navier-Stokes equation (Equation (2)) is solved on the fixed grid to control the motion of multiphase system. The density and the viscosity of the two fluids at any point can be calculated using the two equations given below:

$\rho=\rho_{1}+\left(\rho_{2}-\rho_{1}\right) \tilde{\phi}$

$\eta=\eta_{1}+\left(\eta_{2}-\eta_{1}\right) \widetilde{\phi}$

where $\rho_{1}$ and $\rho_{2}$ are the densities of continuous phase and dispersed phase, and $\eta_{1}$ and $\eta_{2}$ are the viscosities of continuous phase and dispersed phase. A smeared out 
approach is used where the discretisation of the Heaviside function $\left(H_{s m}(\phi)\right)$ can be useful as it is better suited to numerical computations (Deshpande \& Zimmerman, 2006):

$\widetilde{\phi}=H_{s m}(\phi)=\left\{\begin{array}{lc}0, & \text { if } \phi<-\varepsilon \\ \frac{1}{2}+\frac{\phi}{2 \varepsilon}+\frac{1}{2 \pi} \sin \left(\frac{\pi \phi}{\varepsilon}\right), & \text { if }-\varepsilon \leq \phi \leq \varepsilon \\ 1, & \text { if } \phi>\varepsilon\end{array}\right.$

where $\varepsilon$ denotes the interface thickness. The $F_{s t}$ term acting on the interface between two fluid phases is determined by following equation:

$F_{s t}=\sigma k \mathrm{n}_{\Gamma} \delta_{s m}$

where $\sigma$ denotes surface tension, $k$ denotes local interfacial curvature, $\mathbf{n}_{\Gamma}$ is the unit normal vector to the interface pointing into the droplet, and the $\delta_{s m}$ denotes the smeared out Dirac delta function $\left(\delta_{s m}\right)$ concentrated at the interface between two fluids. These above parameters can be calculated by Equation 8, 9, and 10, respectively:

$k=-\nabla \cdot \mathbf{n}_{\Gamma}$

$\mathbf{n}_{\Gamma}=\frac{\nabla \phi}{|\nabla \phi|}$

$\delta_{s m}(\phi)= \begin{cases}0, & \text { if } \phi<-\varepsilon \\ \frac{1}{2 \varepsilon}+\frac{1}{2 \varepsilon} \cos \left(\frac{\pi \phi}{\varepsilon}\right), & \text { if }-\varepsilon \leq \phi \leq \varepsilon \\ 0, & \text { if } \phi>\varepsilon\end{cases}$

To retain the level-set function $(\phi)$, a re-initialization procedure is required for the finite element approximation of the level-set equation. A re-initialized and conservative level-set method is used to describe and convect the fluid interface. The following equation describes the convection of re-initialized level-set function:

$$
\frac{\partial \phi}{\partial t}+\mathbf{u} \cdot \nabla \phi=\gamma \nabla \cdot\left[\varepsilon \nabla \phi-\phi(1-\phi) \frac{\nabla \phi}{|\nabla \phi|}\right]
$$

where $y$ and $\varepsilon$ are numerical stabilization parameters, where the former denotes reinitialization parameter and latter parameter determines the thickness of the interface. Equation (11) is coupled to the governing equations (Equation (2) and (3)) in present numerical model. The $y$ approximates the maximum speed occurring in the computational domain. The $\varepsilon$ assumed as the maximum mesh size in subdomains in the neighbourhood of the interface. After the grid convergence 
analysis, the parameters $Y$ and $\varepsilon$ with the value of $0.065 \mathrm{~m} / \mathrm{s}$ and $5.8 \times 10^{-6} \mathrm{~m}$ were calculated based on the maximum flow velocity in microchannel and optimum mesh size, respectively.

\subsection{Domain Discretisation and Grid Convergence Analysis}

A T-shaped geometry with prescribed dimension of $220 \mu \mathrm{m} \times 90 \mu \mathrm{m}$ was created and meshed with quadrilaterals elements. An entrance thickness $(h)$ of $73.5 \mu \mathrm{m}$ was prescribed in numerical system define the depth of the microchannel. The typical finite element mesh for structured mesh of two-dimension (2D) mapped mesh for a 2D model was selected. Mesh refinement analysis were performed to quantify the dependency of simulation results on the grid size and achieve an optimal grid resolution. Meshes of varying degrees of resolution were set up for the T-junction domain with the same grid size of near-wall region. Table 1 illustrates three examples of mesh geometry with prescribed dimensions in COMSOL Multiphysics. Mesh can be arranged as to be clustered near the wall for optimum grid resolution in order to resolve the boundary layer flow in future work.

Table 1: Comparison of a T-shaped geometry with coarser and finer mesh size.

\begin{tabular}{llllll}
\hline No. of Mesh Elements & 976 (Coarse) & 2072 & 4024 (Finer) \\
\hline 2D Structured Mapped \\
Mesh
\end{tabular}

The present model is set up for transient analysis which provides the time domain response of a system subjected to time-dependent loads. The effect of mesh size was examined by increasing the number of mesh elements from initial number of elements of 976 (coarsest) to 98184 (finest). Finer grid size are generated by simultaneously increasing the number of nodes in all direction to obtain as close to a uniform refinement. The number of elements is increased by a mean factor of $\sqrt{2}$ for each refinement settings. Thus, the total number of nodes for each refinement is 
approximately doubled over the previous grid size. Preliminary test of grid convergence analysis was solely carried out at flow rate ratio, $Q$ of 0.05 , which is a quotient of a flow rate of the flow for dispersed phase and continuous phase. An acceptable relative error and error percentage of $0.15 \%$ and $\leq 1 \%$ between the last two finer grids (12166 and 15963) was obtained, respectively. Fig. 4(b) illustrates the grid convergence analysis at $Q$ of 0.05 . An optimal simulations results was achieved at 7644 number of elements. The relative error of measurements shows the error deviation in relation to the effective droplet diameter between each mesh resolutions. While the error percentage of measurements shows the error deviation in relation to the effective droplet diameter between numerical and experimental data. In the present work, all the parametric studies used, as key output, the effective droplet diameter. Therefore, an integration operator was added to find the area corresponding to the dispersed phase, where $\tilde{\phi} \geq 0.5$, in order to calculate the effective droplet diameter by the following equation:

$d_{e f f}=2 \cdot \sqrt{\frac{1}{\pi} \int_{\Omega}(\tilde{\phi}>0.5) d \Omega}$

This is the diameter of a spherical droplet that has equivalent volume of the formed droplet. The extensive studies of grid convergence analysis was also performed on different flow rate ratio. Fig. 4 illustrates the mesh dependence profile with error percentage of droplet size measurement at different flow rate ratio $Q$. For a constant $Q_{\mathrm{c}}$ at $2.00 \mathrm{ml} / \mathrm{hr}$, the effective droplet diameter was measured with the variation in $Q_{\mathrm{d}}$ $(0.08 \mathrm{ml} / \mathrm{hr}$ to $0.125 \mathrm{ml} / \mathrm{hr})$ for various number of elements. As seen in Fig. 4 , the results of convergence are found to achieve more rapidly and effectively in cases at lower flow rate ratio $(Q \leq 0.0675)$. Higher flow rate ratios are limited to a certain range in numerical model due to difficulties of numerical dissipation in the advection step of fluid simulation. Moreover, further refinement is required to sufficiently resolve the higher velocity profiles. Thus, the subsequent parametric studies were mainly based on $Q$ of 0.05 at optimal mesh size. Particularly, the numerical simulations were performed at a time-step size of $2.57 \times 10^{-5}$ seconds calculated using the Courant-Friedrichs-Lewy (CFL) conditions. It shows a relation between the computational cell size, the transient time-step size, and the fluid velocity within the cell. A Courant number of 0.25 is selected in the present study considering as robust value to maintain the stability of calculations. 
(a)

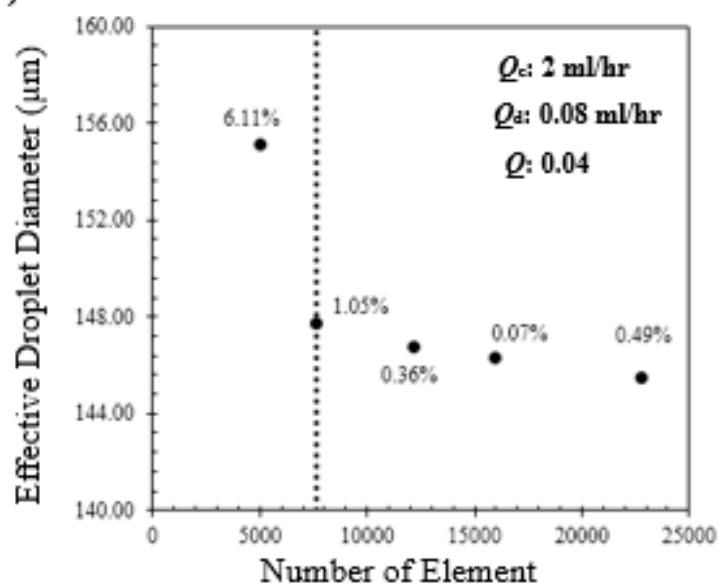

(c)

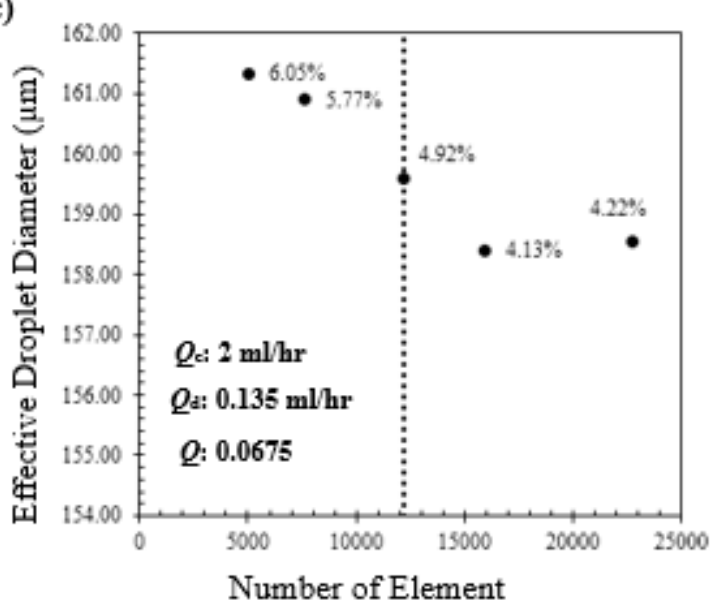

(e)

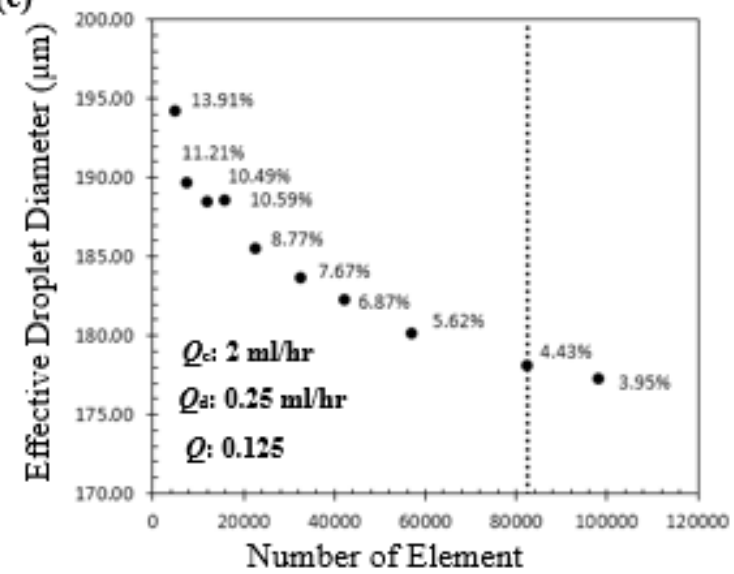

(b)

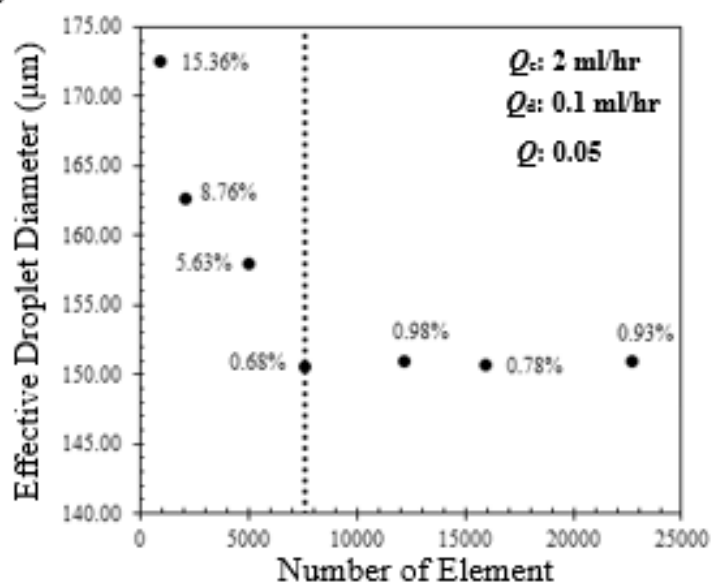

(d)

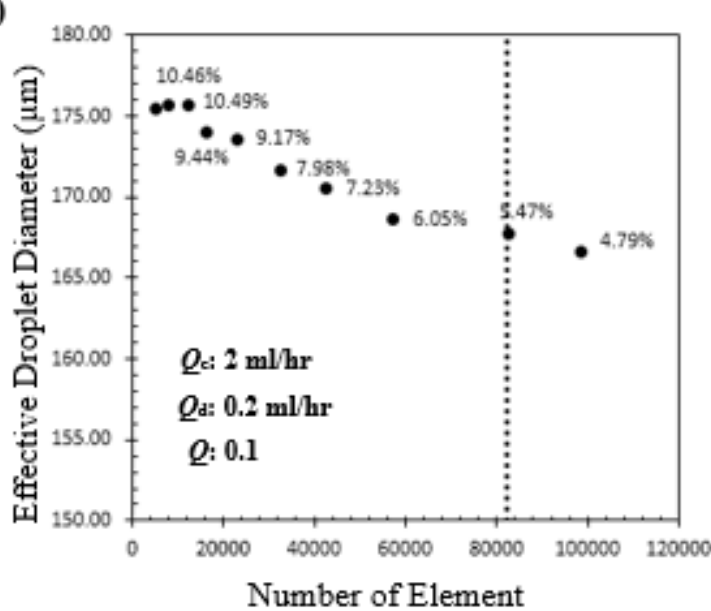

(f)

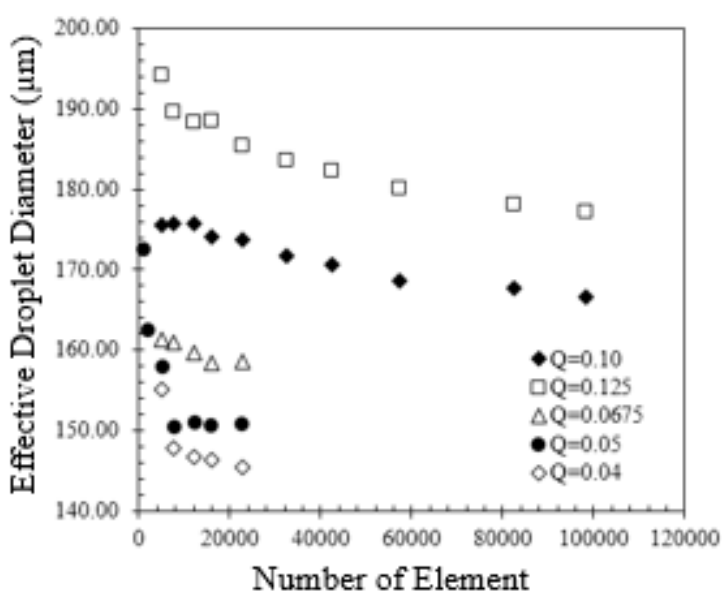

Fig. 4: Grid convergence analysis for different flow-rate ratio profile: (a) $Q=0.04$; (b) $Q=0.05$; (c) $Q=0.0675$; (d) $Q=0.1$; (e) $Q=0.125$; (f) combination of mesh refinement profile.

\subsection{Numerical Model Validation with Experimental Justification}

Mesh convergence analysis was studied to quantify the dependency of simulation results on mesh size and achieve an optimal grid resolution of 7644. A preliminary 
validation of numerical simulations of the formation of deionized water droplets in olive in a T-junction microchannel was carried out and gave qualitative agreement with laboratory experimental data to predict with reasonable accuracy in the range of velocity applied. The results of convergence is found to be achieved more rapidly at lower flow rate ratio. Fig. 5 illustrates the variation in effective droplet diameter between the experimental and numerical studies.

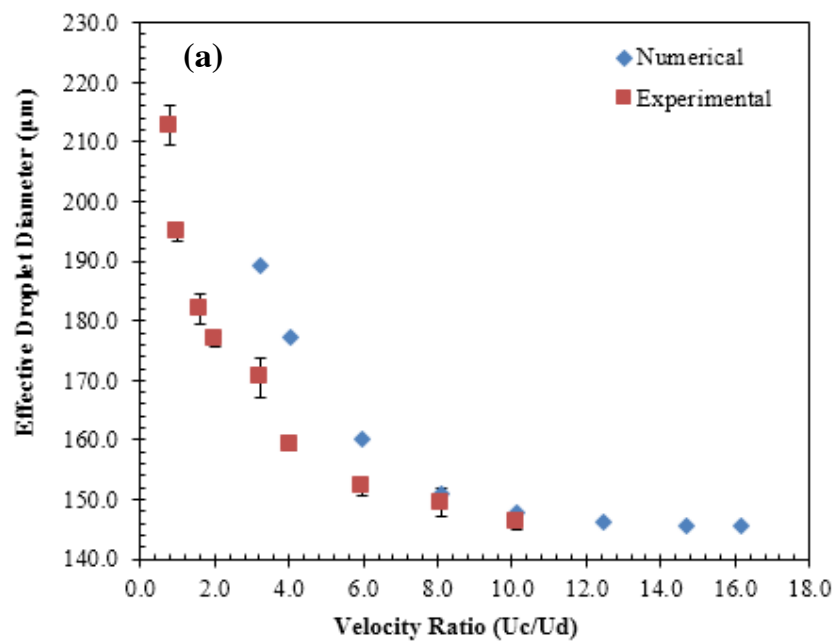

(b)

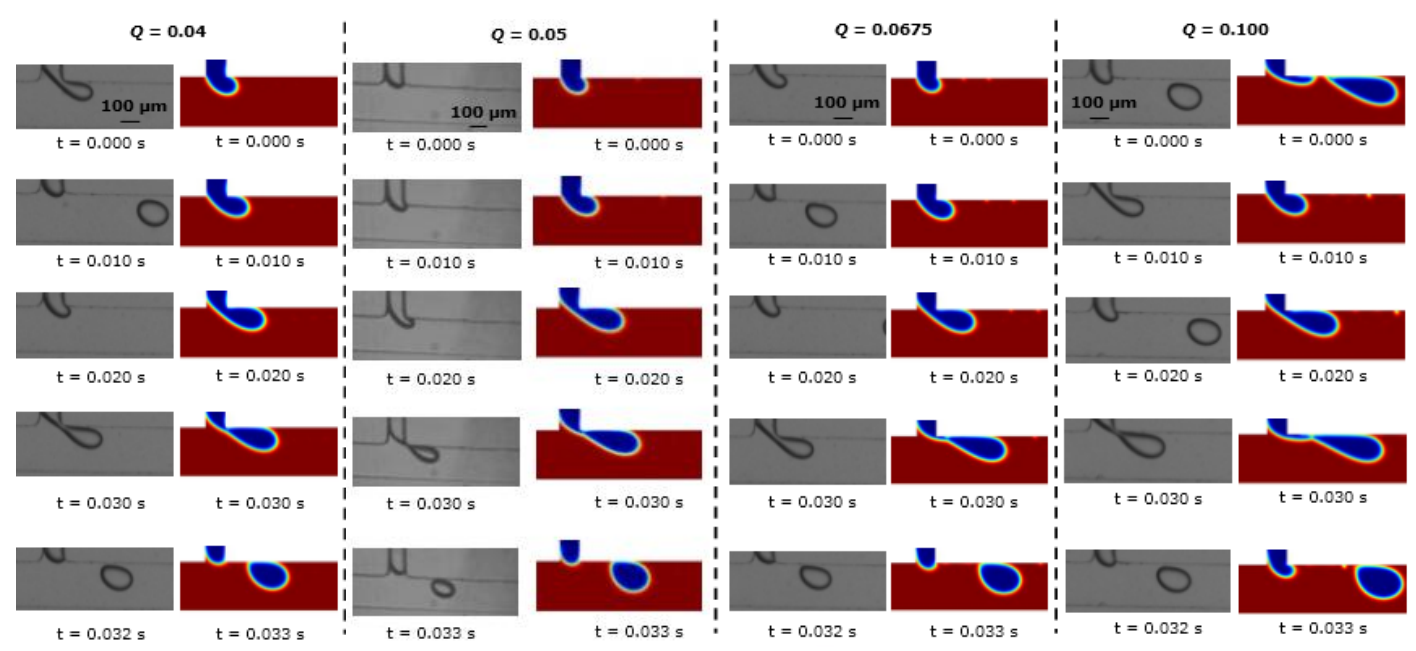

Surface Volume Fraction

Fig. 5: Comparison of (a) effective droplet diameter between numerical and experimental result in the range of velocity ratio applied. Error bars shown indicates the standard deviation in droplet size measurement of 30 droplets under fixed experimental condition. Dashed box shown denotes the (b) droplet breakup phenomena in the range between $Q$ of 0.04 and 0.1 .

The data was recorded at $5 \times 10^{-3}$ seconds intervals to capture the growth and detachment phenomenon of the fluid-fluid interfaces. A contact angle of $180^{\circ}$ that 
represents the complete repulsion of $\mathrm{Na}-\mathrm{CMC}$ droplets by the PDMS with hydrophobic channel wall surface was applied in the computation. As can be seen in Fig. 5, the numerical simulation of detachment process was shown in similar manner with the experimental data at $Q<0.0675$. Nevertheless, such an agreement was physically unreasonable at higher $Q$ as jet breakup phenomena is found to be more

(a)

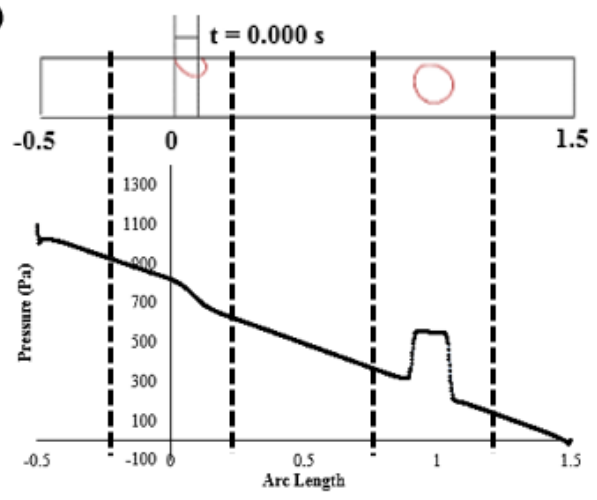

(b)

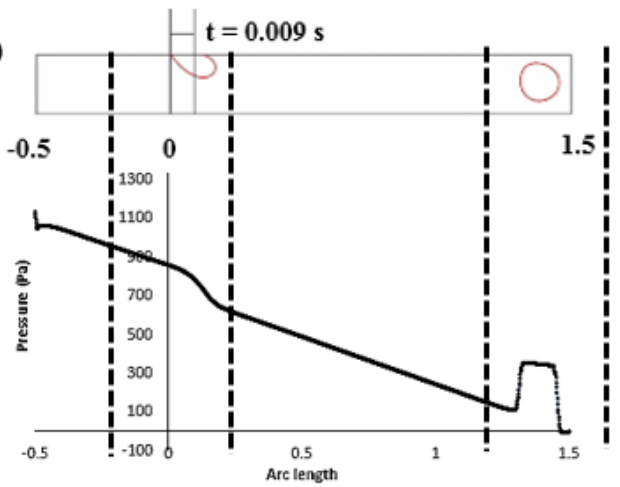

(c)

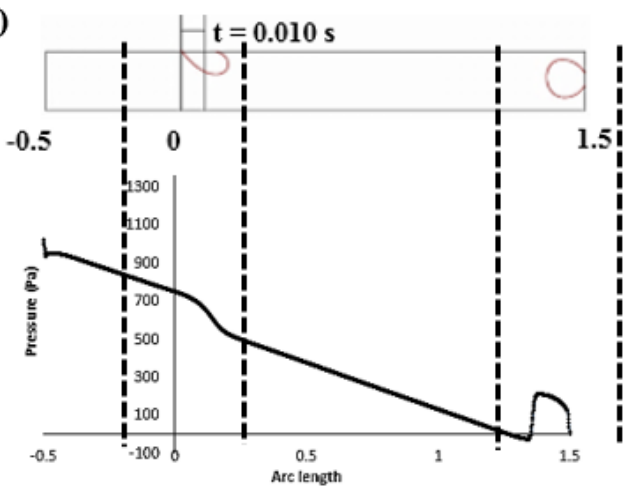

(d)

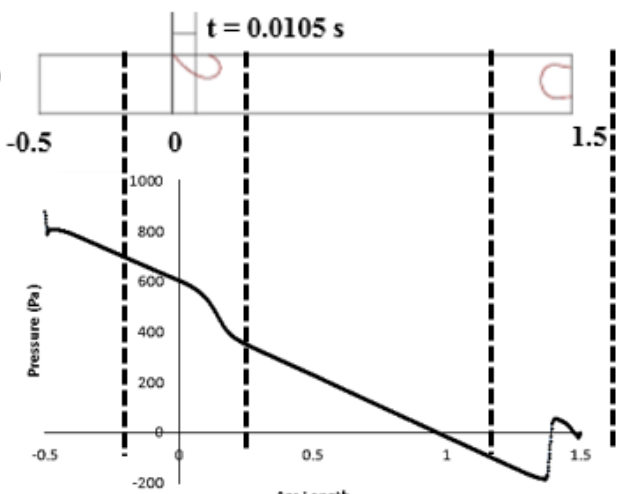

Fig. 6: The pressure distribution of a generated $0.20 \mathrm{wt} \% \mathrm{Na}-\mathrm{CMC}$ droplet interface along the T-junction microchannel at $Q$ of 0.05 .

In the presence of curved interfaces, the curvature induce a pressure jump, which is known as Laplace pressure. The Laplace pressure jumps was determined in the 
path across the droplet body while crossing the front and rear interfaces. As the dimension of the interest gets smaller, surfaces tension becomes dominant over gravitational forces and others physical forces such as viscous and inertial forces. The surface tension becomes an important surface energy parameter that controls the stability of interfaces between the two phases when the droplets are forming. Due to the existence of surface tension effect in a case of a liquid droplet, the Laplace law implies a greater pressure inside the droplet than a continuous phase. As the radius of the droplet become smaller, the pressure becomes larger on the concave side of liquid interface. A Young-Laplace equation is usually used to determine the pressure difference across a fluid interface as a function of curvature. Moreover, the magnitude of this pressure differential can be expressed in term of surface tension:

$$
\Delta P_{L}=\sigma\left(\frac{1}{R_{1}}+\frac{1}{R_{2}}\right)
$$

where $\sigma$ is the surface tension of the respective liquid interface and $R_{1}$ and $R_{2}$ are the two principal curvature radii of the interface. Additionally, the effect of viscous dissipation inside the droplets may also prominently affects the pressure drop distribution when the viscous effect is considerably increased.

\section{Results and Discussion}

\subsection{Effect of Na-CMC Viscosity on Droplet Breakup Time and Production Rate}

The present investigation was designed to determine the Na-CMC viscosity effect on the droplet breakup time and production rate. The viscosity of polymer solution is a function of concentration and the molecular weight of dissolved polymer. While the concentration of $\mathrm{Na}-\mathrm{CMC}$ solution is increased in a series of simulations from 0.02 wt $\%$ to $1.20 \mathrm{wt} \%$ at a constant $Q$ of 0.05 , the evolution of droplet breakup time can be discerned in two distinct non-monotonic behaviour. Fig. 7(a) illustrates the Na$\mathrm{CMC}$ concentration effect on the normalized droplet breakup time whereas the normalized production rate is shown in Fig. 7(b). The variation of viscosity of a shear thinning drop occurs during the pinch off process. Initially, the breakup time decreases as $\mathrm{Na}-\mathrm{CMC}$ concentration increases from $0.02 \mathrm{wt} \%$ to $0.40 \mathrm{wt} \%$. The shear-thinning effect of $\mathrm{Na}-\mathrm{CMC}$ solution increases with the concentration. The greater shear-thinning effect may exhibit a decrease in polymer viscosity upon the 
application of shear near the channel wall due to the inertial force. At concentrations well above $0.40 \mathrm{wt} \%$, the $\mathrm{Na}-\mathrm{CMC}$ droplet breakup time increases may to the viscous force becomes significantly prevailing over the inertial force induced by the continuous phase on the forming interface. In general, the largest shear rate occurs at the corner edge of the T-junction and thus the shear-induced destabilization of the dispersed thread causes the breakup of thread leading to the formation of droplets. As can be seen in Fig. 8, the distribution of non-Newtonian wall shear-rate decreases as compared to the Newtonian wall shear rate profile. While in the lower wall shear rate range, the $\mathrm{Na}-\mathrm{CMC}$ solutions exhibited a significant increase in viscosity. This means the low shear rate viscosity occurs at the high concentration of Na-CMC content. Consequently, the droplets pinch-off in larger viscosity fluids for which the inertial effects are unamplified.

(a)
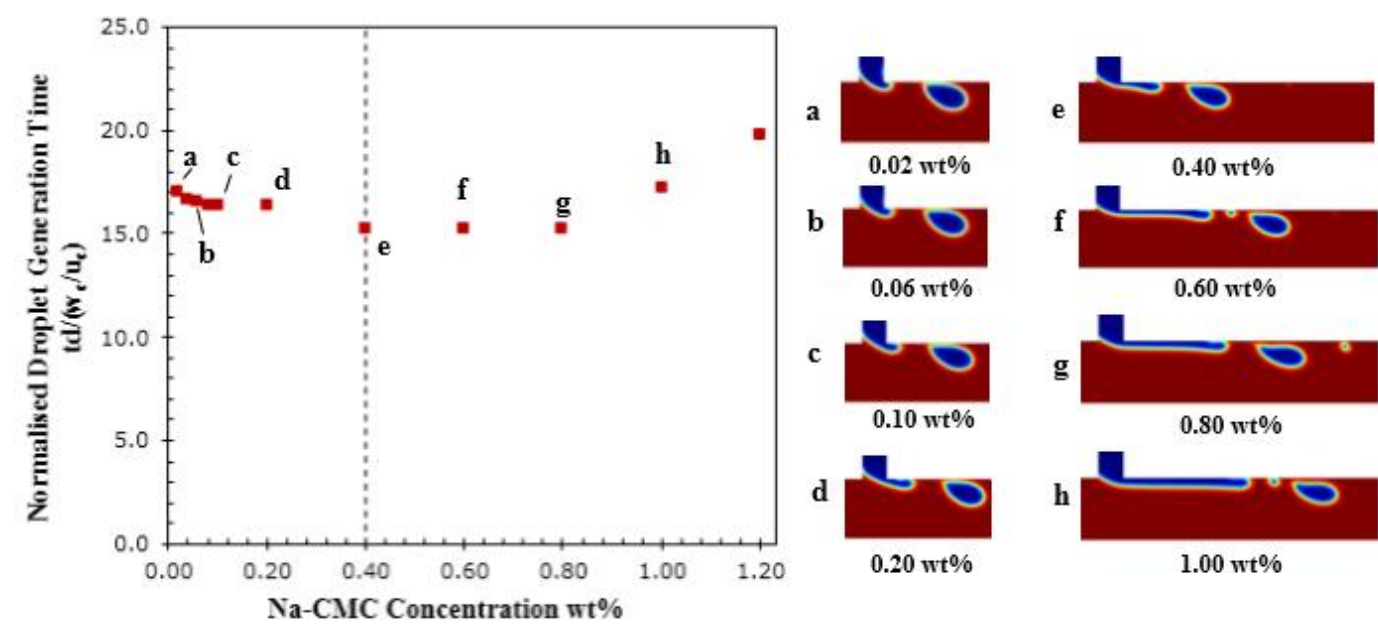

$0.40 \mathrm{wt} \%$
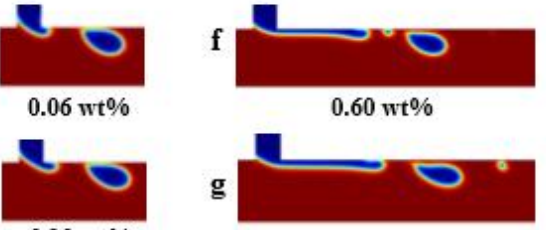

$0.60 \mathrm{wt} \%$

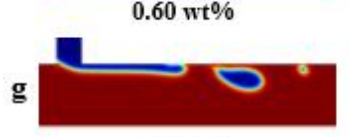

$0.80 \mathrm{wt} \%$
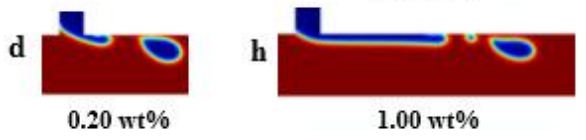

406

(b)

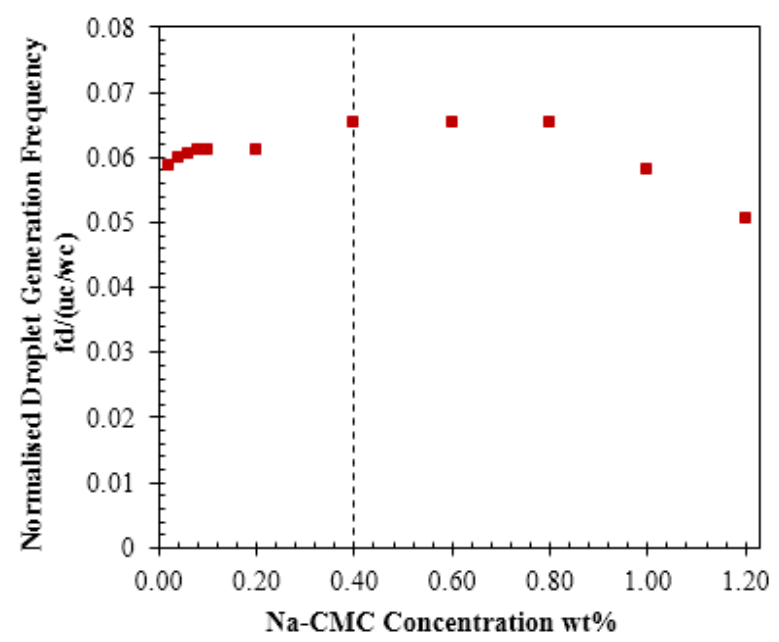

Fig. 7: Effect of Na-CMC concentration on (a) droplet breakup time (b) droplet 409 production rate (for system: $Q_{\mathrm{d}} / Q_{\mathrm{c}}=0.05$ ). 
At dilute $\mathrm{Na}-\mathrm{CMC}$ concentrations below 0.40 wt $\%$, the viscosity effect is not considerable as the direct intermolecular interactions are negligible. As the Na-CMC concentration increases, the shear-thinning effect becomes more significant and causes rapid pinch-off due to the high shear stress exerted by the continuous phase near to the wall. The viscosity of the shear thinning drop is reduced when the drop begins to neck response to the increased shear rate in that region. As the neck continues to thin, the region of lower viscosity grows to encompass almost the entire drop occurring within the neck and just outside it where the outflows from the neck occur (Malcolm R. Davidson \& Cooper-White, 2006; M. R. Davidson, Cooper-White, \& Tirtaatmadja, 2004). Nevertheless, extending breakup occurs and lower production rate was found for the $\mathrm{Na}-\mathrm{CMC}$ solution concentration above $0.40 \mathrm{wt} \%$. This is mainly caused by the development of entanglement coupling between the polymer chains, which begins manipulating the fluid characteristics of $\mathrm{Na}-\mathrm{CMC}$ solution. The elongation of the necking into a thin filament connect between the primary droplet and the upper dispersed phase thread, suggest that an increase in polymer concentration which plays an important role in resisting drop pinch-off with the formation of thinning filament. Thus, the viscous fluid thread is then stretched by the mainstream flow to the downstream region and the breakup event is delayed substantially. 

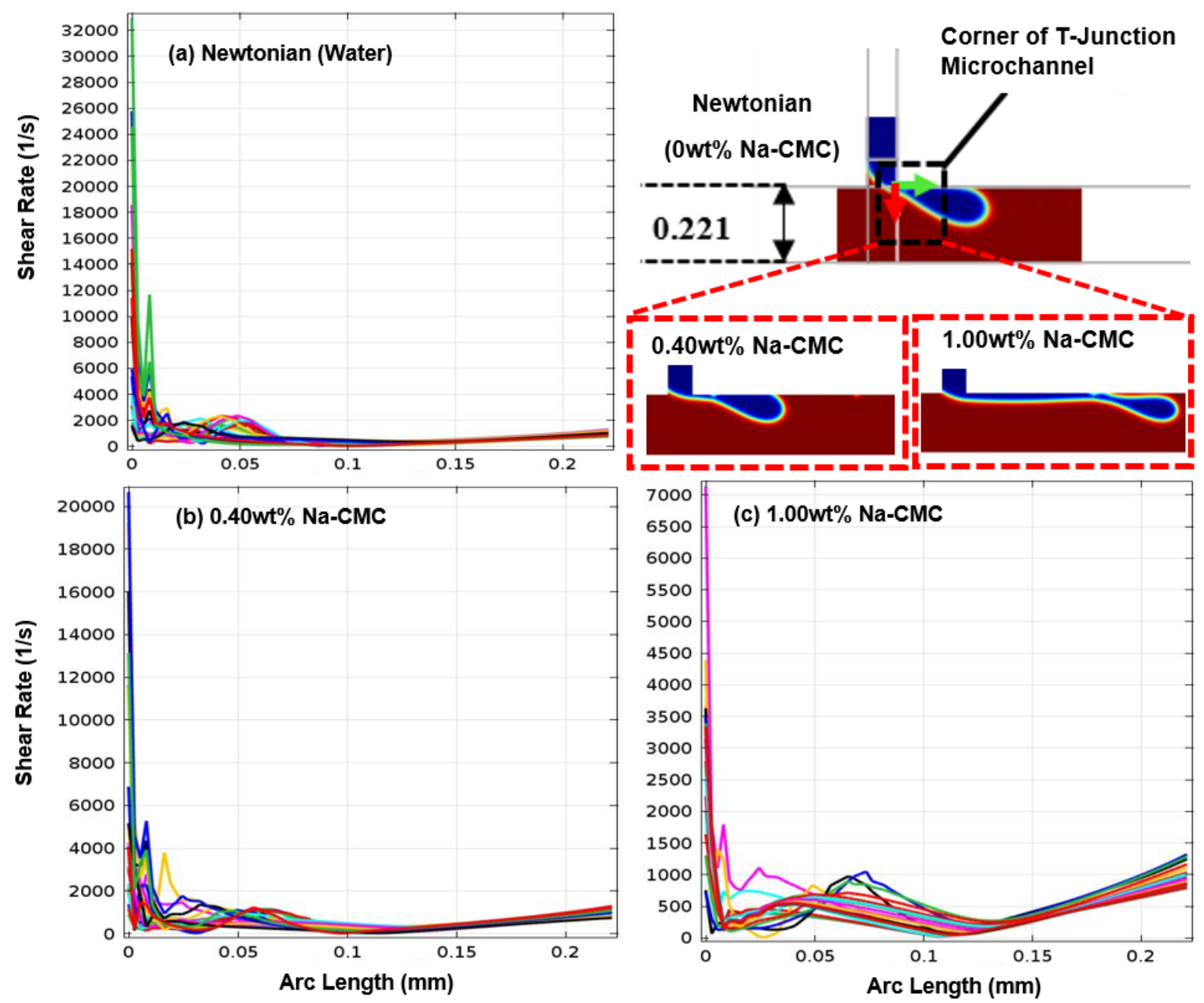

Fig. 8: Shear rate profile of fluid flow at the edge of T-junction microchannel along the arc length of continuous phase for (a) Newtonian system; (b) $0.40 \mathrm{wt} \% \mathrm{Na}-\mathrm{CMC}$ non-Newtonian system; and (c) $1.0 \mathrm{wt} \% \mathrm{Na}-\mathrm{CMC}$ non-Newtonian system (for system: $\left.Q_{\mathrm{d}} / Q_{\mathrm{c}}=0.05\right)$.

Fig. 9 illustrates the Laplace pressure profile of a $\mathrm{Na}-\mathrm{CMC}$ droplet interface curvature for different polymer concentrations at the middle plane of the microchannel. At dilute $\mathrm{Na}-\mathrm{CMC}$ concentration below $0.40 \mathrm{wt} \%$, droplets are selfpropelled some distances from the wall mainly subjected to the gradient of the surface energy and higher velocity flow stream of the main channel after the sharp breakup occurs at the corner of T-junction. For the larger concentration of $\mathrm{Na}-\mathrm{CMC}$ (C > $0.40 \mathrm{wt} \%$ ) dispersed fluid, the presence of instabilities promotes the breakup of jets. After the formation of the primary breakup droplets, the generated droplet moves near the wall of microchannel as it is initially exposed to the flow projected from the side branch at the moment of breakup. After a certain distance, it will flow near the center of the bulk phase. The droplet with a radius in an emulsion will exert greater pressure on the inner concave interface than on the convex side. When the 
droplet deformation occurs, the Laplace pressure of the deformed droplet is a function of the radius along the droplet surface (see section 3.4). Thus, as the dispersed thread is elongated, a smaller droplet will be formed causing smaller radius of curvature that result in a larger inward force and expected to experience a greater pressure (see Fig. 9).

(a) $0.10 w t \%$

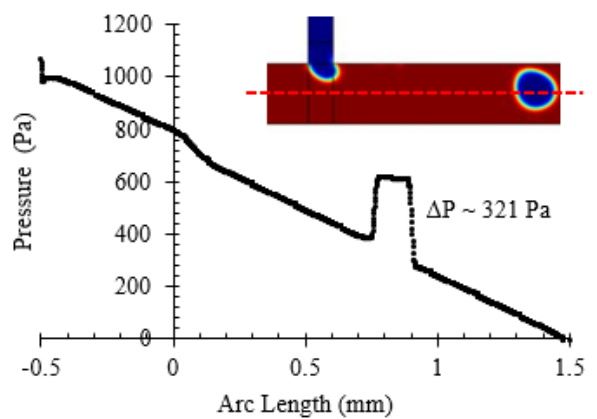

(c) $0.80 w t \%$

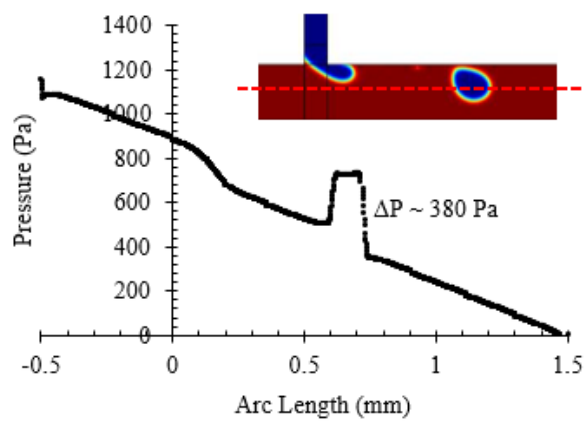

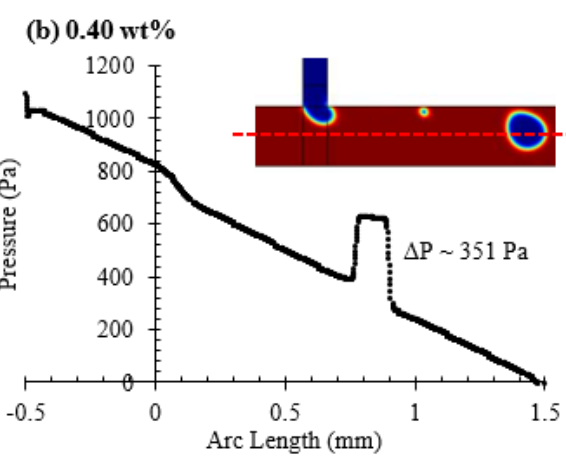

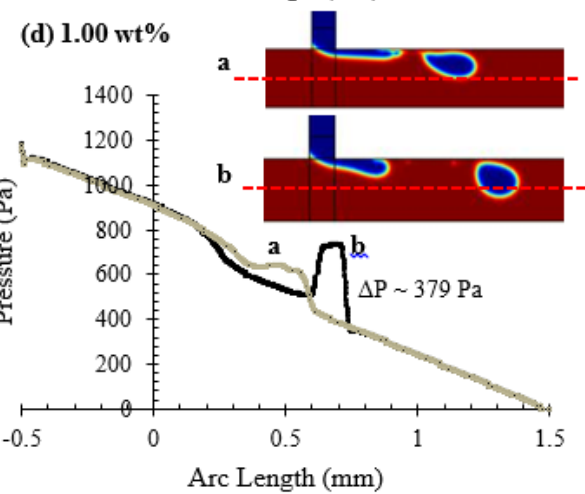

Fig. 9: A qualitative plot of the Laplace pressure profile of a generated $\mathrm{Na}-\mathrm{CMC}$ droplet interface curvature at concentration of $0.10 \mathrm{wt} \%, 0.40 \mathrm{wt} \%, 0.80 \mathrm{wt} \%$, and $1.00 \mathrm{wt} \%$ along the middle plane of microchannel (for system: $Q_{\mathrm{d}} / Q_{\mathrm{c}}=0.05$ ).

\subsection{Effect of Surface Wettability on Droplet Breakup Time and Production Rate}

The effect of shear-thinning nature on droplet breakup process has not been extensively studied in the preceding analysis. In a T-shaped microchannel, the continuous and dispersed phase were dispensed at the prescribed flow rates through the microchannel with a hydrophobic wall surface $\left(\theta>90^{\circ}\right)$. Additionally, the continuous phase needs to wet the surface of the channel walls preferentially in order to repel the dispersed phase droplets away from the wall. The surface wettability of microchannel are of utmost importance for the stability of the droplets formation process in a microfluidic device. The effect of surface wettability on the total droplet formation time, which is the transition between a growing and a detached droplet, was discussed. Fig. 10 illustrates surface wettability effect on the 
470 normalized droplet formation rate. Results revealed that the droplet detachment 471 occurs more rapidly at shorter times as the $\theta$ increases. The inertial force, shear force and surface wettability are competing effects influencing the dynamics of the droplet breakup process. The wettability driving the fluid toward the surface and the contact area between the droplets and solid surface increases for the smaller $\theta$. The smaller $\theta$ tends to reduce the droplet deformation and delay the breakup process. As $\theta$ increases, the thread is no longer flowing close to the wall as its adhesion strength to the wall reduces, causing less resistance to flow. Therefore, the inertial force driving the flow of thread is prevailing. The time for the formation of droplet is also essentially dependent of the strength of shear force acting on the interface, because it dominates over the adhesion force for larger $\theta$. Bashir et al. (2011) reported that the degree of confinement promotes the breakup for the larger $\theta$; whereas the degree of confinement suppresses the breakup for smaller $\theta$. 

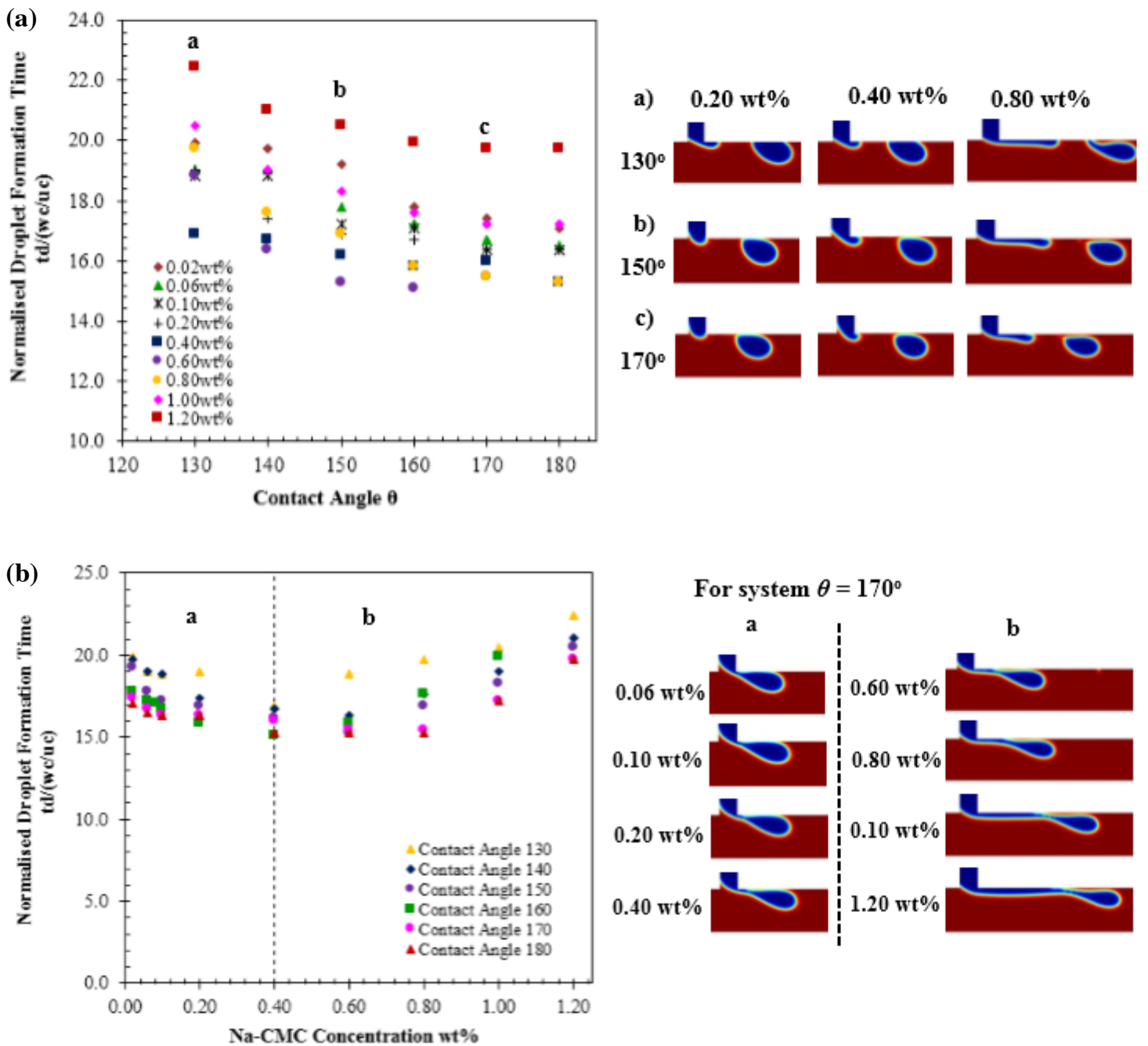

501
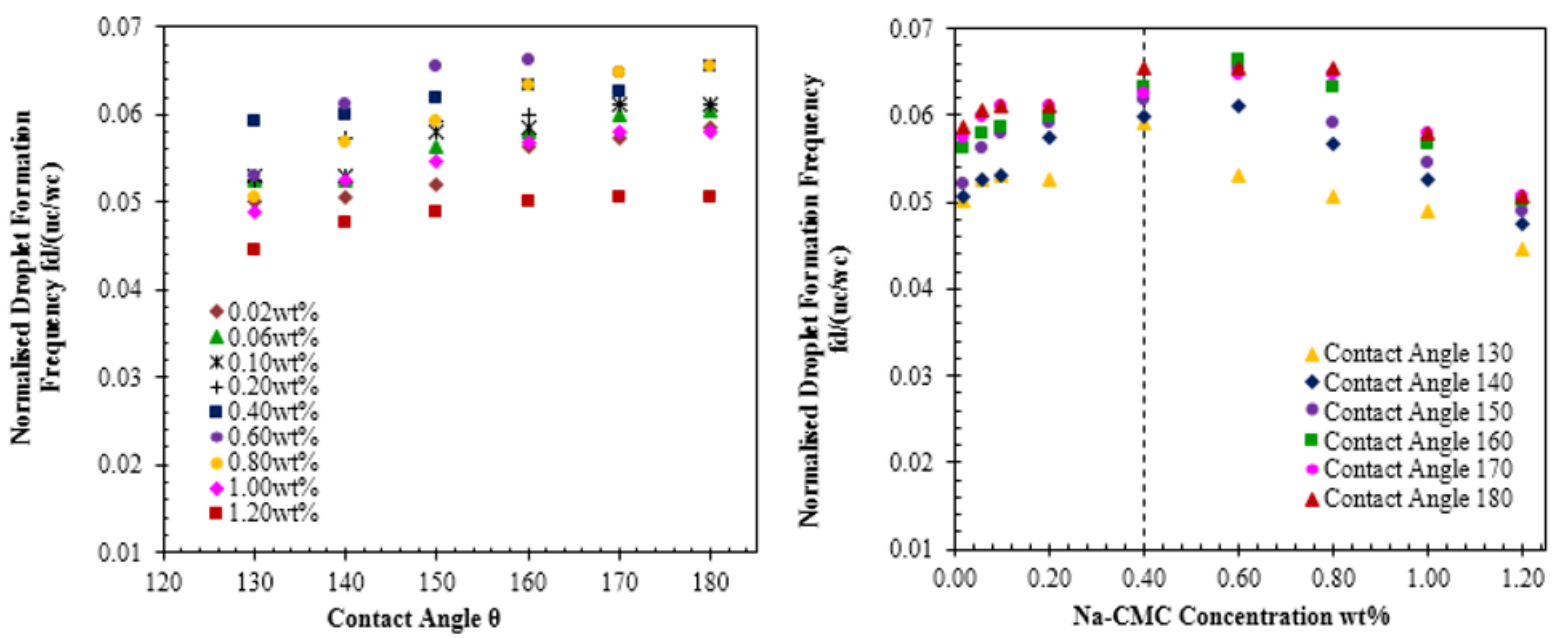

Fig. 10: (a) Effect of contact angle on the normalized droplet formation time of NaCMC solutions. (b) Variation in normalized droplet formation rate of shear-thinning droplets for a. dilute $(C<0.40 \mathrm{wt} \%)$ and b. semi-dilute $(\mathrm{C}>0.40 \mathrm{wt} \%) \mathrm{Na}-\mathrm{CMC}$ concentrations regime at fixed $\theta$ (for system: $Q_{\mathrm{d}} / Q_{\mathrm{c}}=0.05$ ). 
For a fixed value of $\theta$, the result reveals that the droplet formation time decreases as the concentration of the Na-CMC increases from 0.02 wt\% to $0.40 w t \%$ (see Figure 10). Interestingly, a similar phenomenon does not happen for the larger concentration $(C>0.40 w t \%)$. This is due to the fact that the concentration of the polymer solutions beyond the critical overlap concentration exert a significant

Hong and Cooper-White (2009) studied the effect of non-Newtonian Carbopol dispersions on the droplet detachment behaviour in the bulk phase of silicone oil. The Carbopol droplet pinch-off time increases with increasing the viscosity of dispersions ${ }^{37}$. Besides, Arratia et al.(2009) also reported that the time for polymer polyacrylamide (PAA) droplet breakup was retarded as the polymer molecular weight is increased at fixed $Q$ (Hong \& Cooper-White, 2009). Husny and Cooper-White (2006) also claimed that pinch-off occurred rapidly without any significant filament formation during necking for Newtonian droplet formation; but, this rapid necking event was retarded with the formation of a thinning filament for polyethylene oxide (PEO) solutions (Husny \& Cooper-White, 2006). As can be seen in Fig. 10, a similar observation was found for the higher Na-CMC concentration as the formation of a thin and stable filament between the droplet and thread is more apparent when $\mathrm{C}>$ $0.40 \mathrm{wt} \%$. The dynamics of the thinning filament are governed by the shear stress and viscous pressure, by which the filament is elongated drastically and resists the droplet pinch-off. Thus, the delayed pinch-off may be due to the retardation effect of the fluid elasticity, which it can be increased by increasing the polymer concentration. However, the entire phenomena was observed from our predictive model with no elastic stress applied in our present study. Hence, that shear-thinning alone is also sufficient to induce a filament and that elasticity is not necessary. 
541 In contrast, results revealed that the production rate of the $\mathrm{Na}$-CMC droplet increases with enhanced surface wettability. The droplet generation frequency increases with dispersed phase concentration over the range of $0.02 \mathrm{wt} \%$ to 0.40 $\mathrm{wt} \%$ when the contact angle was held constant at each wetting condition. However, the rate of droplet production begins to decrease with increasing the concentration of $\mathrm{Na}-\mathrm{CMC}$ dispersed phase concentration larger than $0.40 \mathrm{wt} \%$, as noted in the previous section.

A comparison of pressure profiles of $\mathrm{Na}-\mathrm{CMC}$ droplet at different contributions were illustrated in Fig. 11. At low and dilute concentrations of the Na-CMC polymer, the pressure drop of a stabilized curved surface of $\mathrm{Na}-\mathrm{CMC}$ droplet is increasing along the bulk phase with increasing the wetting conditions. The weak adhesion and strong cohesion of the liquid in the bulk phase was found for the larger contact angles. The unfavourability of channel surface to the dispersed thread plays and leads a major role in the transport of droplet and lead to a larger shear force to be exerted on the drop surface. Rapid deformation of the droplet occurs due to the adequate shearing force induced by the continuous phase. Thus, the droplet mass was shown to decrease when the initial contact angle is larger and leads to a larger pressure drop. The higher concentration of $\mathrm{Na}-\mathrm{CMC}$ dispersed phase contributes the same phenomenon. For the $\mathrm{Na}-\mathrm{CMC}$ concentration above $0.40 \mathrm{wt} \%$, lower contact angles promote and dampen greater spreading and dampens the breakup process. The spreading of the elongated thread is eventually broken up into smaller droplets when the surface energy is overcome. Neglecting the impact of fluid property on surface wettability, the much larger concentration of fluid samples withdrawn from the dispersed thread can create much greater pressure drop at the same contact angle. 

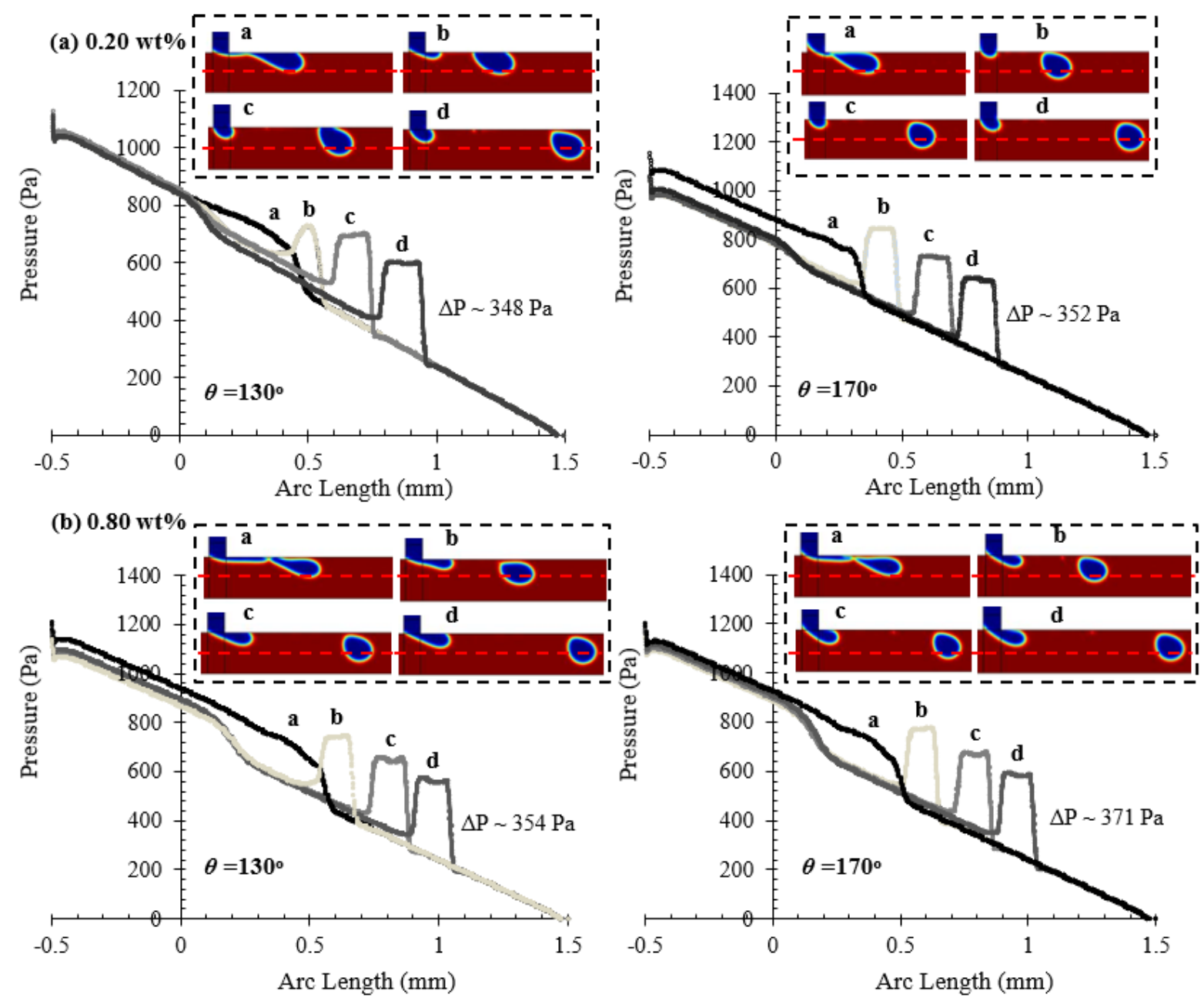

Fig. 11: A qualitative plot of the Laplace pressure profile of a generated $\mathrm{Na}-\mathrm{CMC}$ droplet interface curvature at concentration of $0.20 \mathrm{wt} \%$ and $0.80 \mathrm{wt} \%$, along the middle plane of microchannel at $\theta=130^{\circ}$ and $170^{\circ}$ (for system: $Q_{d} / Q_{c}=0.05$ ).

\subsection{Effect of Interfacial Tensions on Droplet Breakup Time and Production Rate}

The effect of interfacial tension on the time taken for the droplet growth, deformation and detachment were also investigated. The impact of interfacial tension on the $\mathrm{Na}-$ CMC droplet breakup time is illustrated in Fig. 12(a). As a result, the droplet breakup time increases with increasing interfacial tension. As interfacial tension increases, the retraction of the interface induced by surface tension forces becomes greater due to the relatively high surface free energy. This will tend to hinder the droplet formation process as the Na-CMC droplets take longer time approach to thermodynamics equilibrium. At the low concentration regime $(C<0.40 \mathrm{wt} \%)$, the droplet is pinched-off sharply at the corner of T-junction. In addition, this breakup 
regime is driven primarily by the build-up of pressure upstream which is mainly due to the high degree of confinement of the droplet in bulk phase.
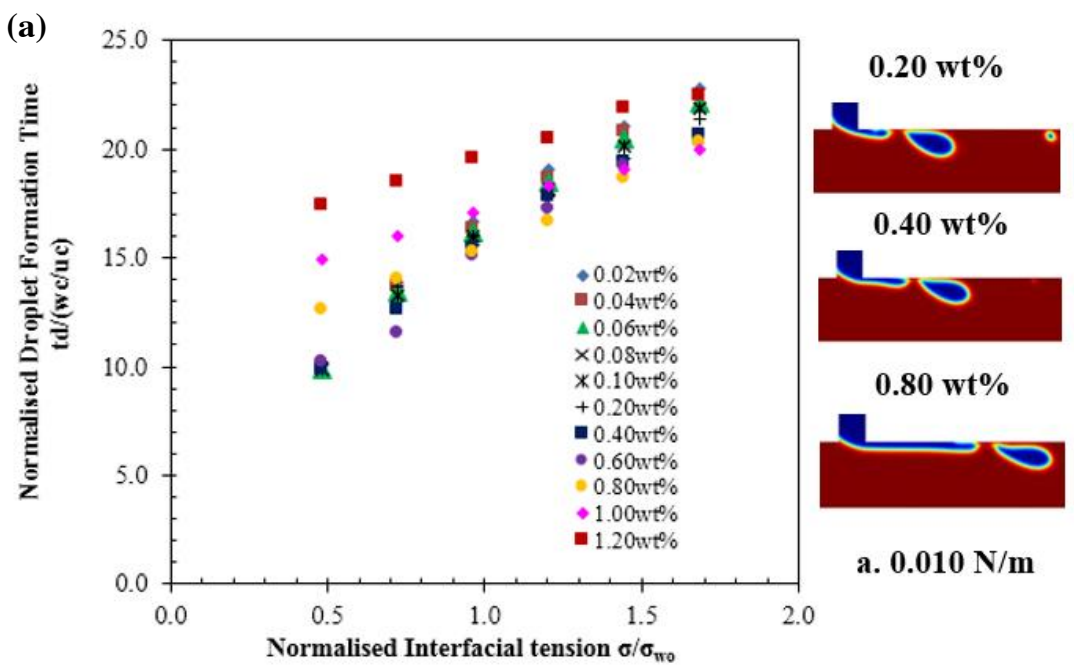

0.20 wt $\%$ $0.20 \mathrm{wt} \%$

(b)

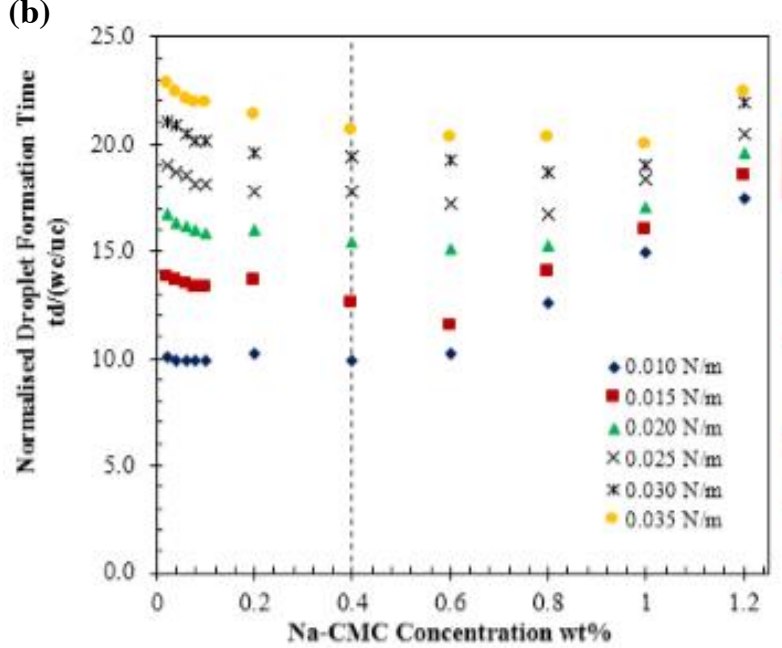

For system: $\sigma=0.010 \mathrm{~N} / \mathrm{m}$
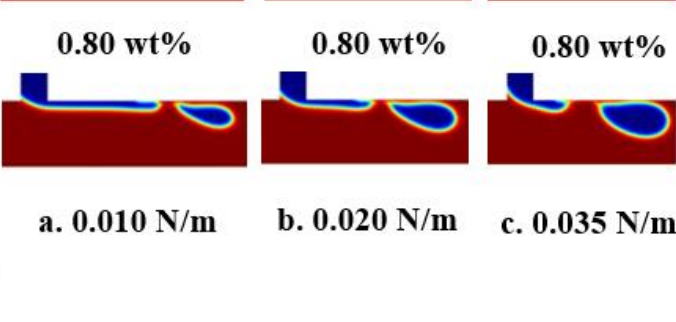

a. $0.010 \mathrm{~N} / \mathrm{m}$

b. $0.020 \mathrm{~N} / \mathrm{m}$

c. $0.035 \mathrm{~N} / \mathrm{m}$
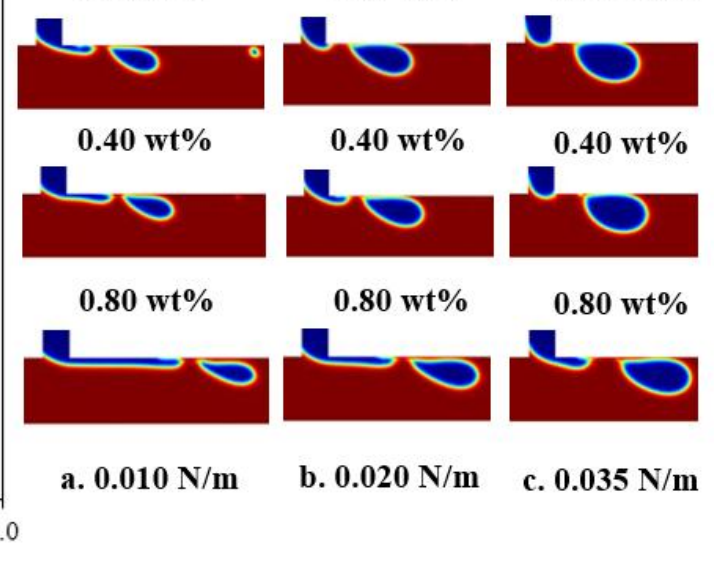

586
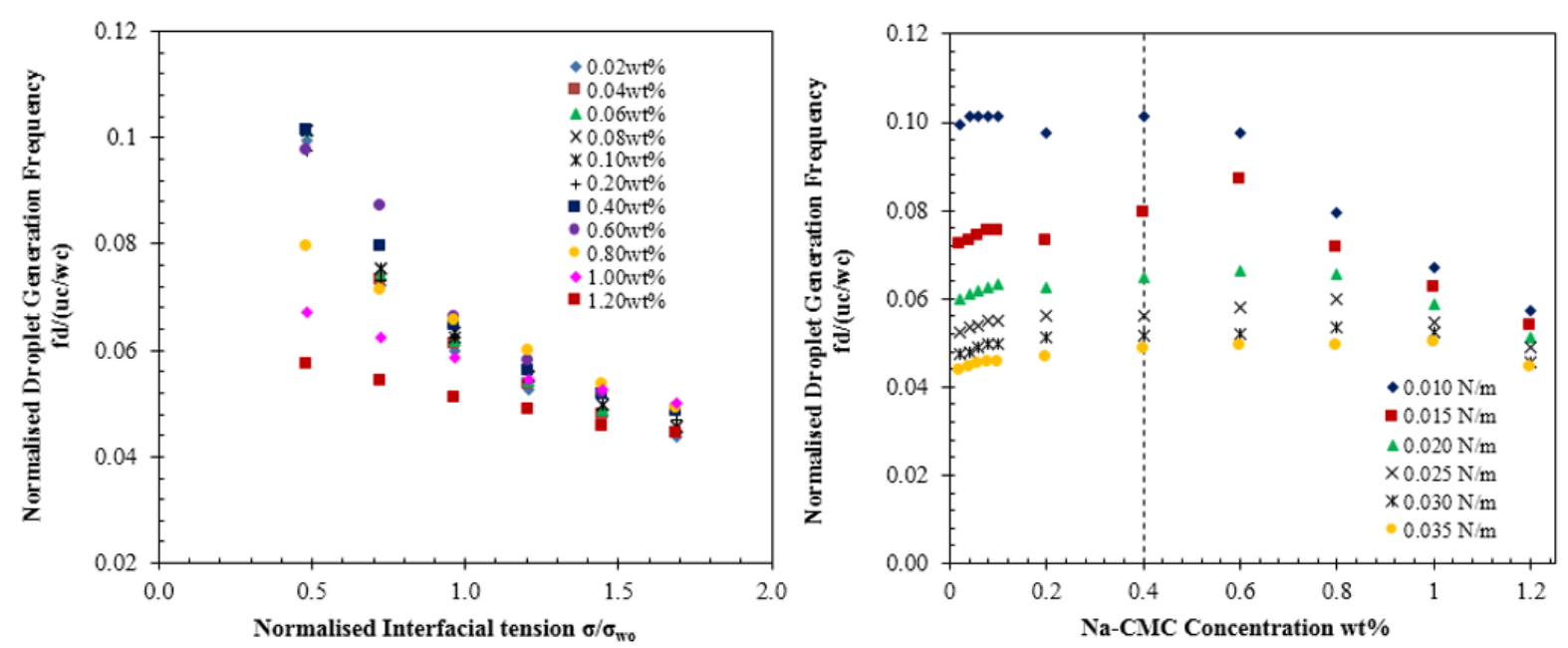

Fig. 12: (a) Effect of interfacial tension on the normalized droplet formation time of 
formation rate of $\mathrm{Na}-\mathrm{CMC}$ droplets for various concentrations at each interfacial tension $(\sigma)$.

The droplet breakup time decreases when $\mathrm{Na}-\mathrm{CMC}$ concentration is increased from $0.02 \mathrm{wt} \%$ to $0.40 \mathrm{wt} \%$ (see Figure $12(\mathrm{~b})$ ). In contrast to this phenomenon, the breakup time increases as $\mathrm{Na}-\mathrm{CMC}$ concentration is larger than $0.40 \mathrm{wt} \%$. For dilute $\mathrm{Na}$ $\mathrm{CMC}$ concentrations below $0.40 \mathrm{wt} \%$, the interfacial forces are more dominant than viscous forces due to the insufficient polymer chain overlap leading to earlier occurrence of pinch-off. While the Na-CMC concentrations above $0.40 \mathrm{wt} \%$, interfacial forces are less prevalent in strength and the larger viscosities give rise to longer breakup time which can be attributed to the higher magnitude of the dispersed thread pressure. A similar observation has been reported by Zhang and Basaran (1995) who studied the high viscous pendant drops (Zhang \& Basaran, 1995). Tirtaatmadja et al. (2006) also claimed that the polymer molecules can be highly extended during their approach to pinch region and this contributes to the formation of filament. The filament can be further extended by the stretching force at a constant rate until full extension of polymer coil is achieved (Tirtaatmadja, McKinley, \& Cooper-White, 2006). In general, high stretching of polymer chains is associated with high elasticity.

For simplicity the viscous force is disregarded, the jetting phenomena and the prolonged thinning of the fluid filament at the rear is more substantial for the shearthinning droplet with lower interfacial tension at dilute concentration regimes. Less energy is required to disrupt an interface with low magnitude of interfacial tension. Thus, low interfacial tension liquid thread tends to breakup rapidly. Nevertheless, at low $Q$, jetting occurs when the inertial forces induced by continuous phase exceed interfacial tension forces. As noted in the previous observation, the length of filament gets longer when the Na-CMC polymer concentration is increased. This presumably prevents the neck of the dispersed thread from pinching off. Thus, the existence of a thin polymeric filament will tend to decelerate the breakup process, especially for $\mathrm{C}>$ $0.40 \mathrm{wt} \%$. Previous studies have reported that the formation of filament was due to the elasticity effect. Nevertheless, a similar behaviour was also found for the working solution which is purely viscous and shear-thinning characteristics at the larger concentrations. 
624 The breakup time for a droplet is increased leading to a decreased production rate at fixed $Q$ for larger magnitude of interfacial tension forces. This exhibits the similar observations to the studies made by Peng et al. (2011) and Bashir et al. (2011) who studied water-oil emulsions in flow-focusing and cross-flowing microfluidics devices, respectively. The droplet production rate at each fixed interfacial tension has been investigated. In lower concentration regimes ( $C<0.40 \mathrm{wt} \%$ ), the earlier occurrence of droplet breakup is observed. In contrast, the production rate decreases with increasing the $\mathrm{Na}-\mathrm{CMC}$ concentration in the semi-dilute concentration regime ( $\mathrm{C}>$ $0.40 \mathrm{wt} \%)$, it takes a longer time for the dispersed phase viscous force to be overcome by the opposing inertial force and shear stress induced by the continuous phase.

The pressure-jump profiles of $\mathrm{Na}-\mathrm{CMC}$ droplet at different interfacial tensions were illustrated in Fig. 13. At lower concentration of $\mathrm{Na}-\mathrm{CMC}$ solutions, the larger interfacial tension contributes to a larger pressure drop profile. As interfacial tension increases, the strength of attractive force increases and causes the liquid surface to contract toward the interior phase and thus the repulsive collisional forces is reduced in order to resist the contraction. Thus, the breakup process of droplet is hindered and larger droplet is generated. Similarly, fluids with the higher concentration produce larger size droplets at the larger surface tensions. Nevertheless, the generation of smaller droplets did not reveal a larger pressure-drop profiles when compare to the previous consequences. It is postulated that the effect of surface tension enables higher surface energy which is caused by the gradient of confinement. Forcing the detached droplet translocate through a confined region will increase the pressure within the droplet at the location where it is almost fully occupied. This causes the increment of the curvature effect that requires the external fluid to apply the extra pressure. 

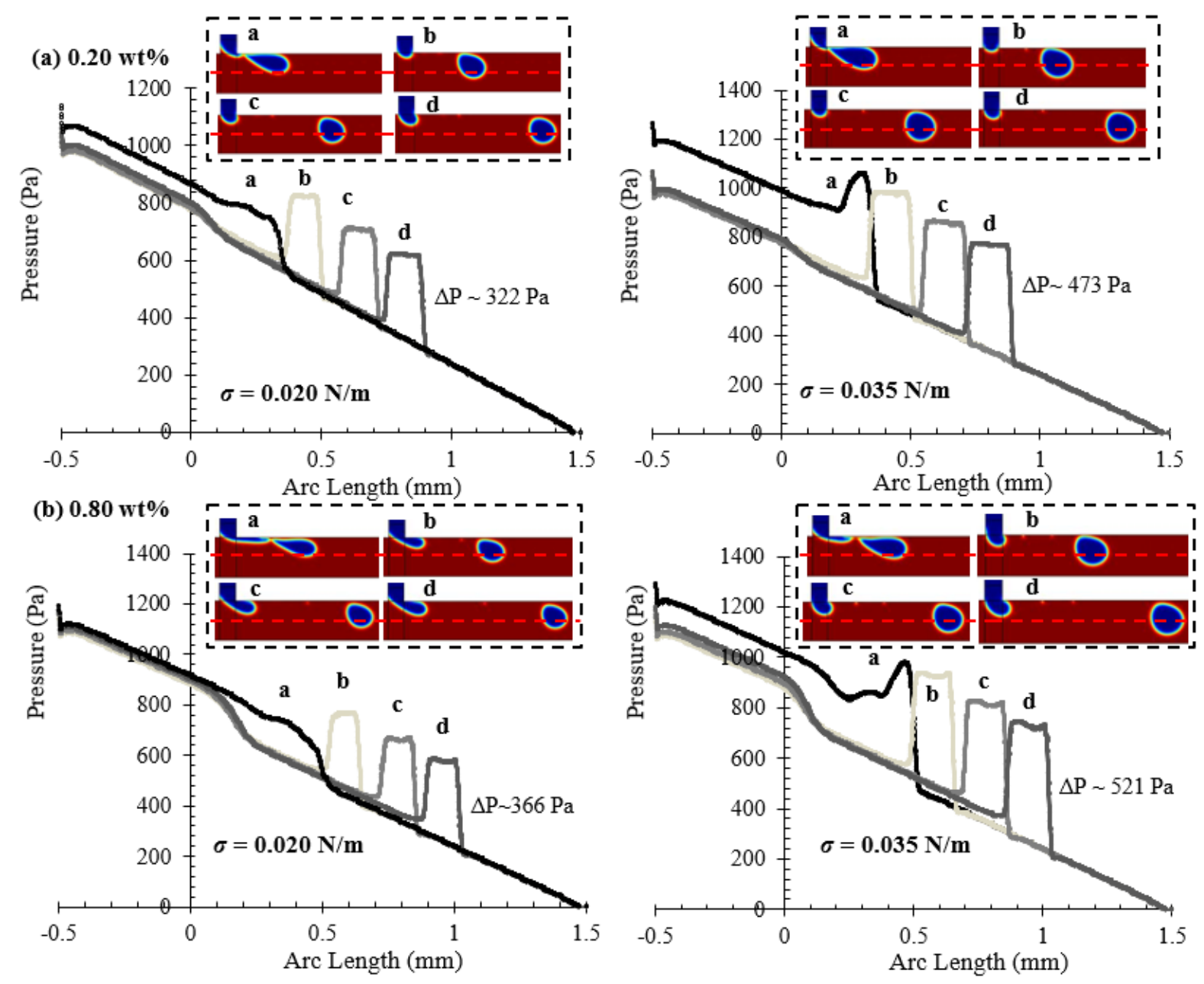

Fig. 13: A qualitative plot of the Laplace pressure profile of a generated Na-CMC droplet interface curvature at concentration of $0.20 \mathrm{wt} \%$ and $0.80 \mathrm{wt} \%$ along the middle plane of microchannel at $\sigma=130^{\circ}$ and $170^{\circ}$ (for system: $Q_{d} / Q_{c}=0.05$ ).

\section{Conclusions}

The essential role of viscosity, surface wettability, and interfacial tension on emulsification process of $\mathrm{Na}-\mathrm{CMC}$ shear-thinning droplet has been highlighted in the present simulation using conservative level-set numerical method. The evolution of the breakup time and the droplet production rate is effectively governed by the physical properties of working fluid. Olive oil was selected as the continuous phase while the Na-CMC polymer was used as the non-Newtonian dispersed phase fluid. The present simulation data revealed that droplet breakup time and production rate have a striking non-monotonic relationship with the $\mathrm{Na}-\mathrm{CMC}$ polymer concentration due to the considerable rheological shear-thinning nature of $\mathrm{Na}-\mathrm{CMC}$ polymer solution. As the concentration increases, the polymer concentration crosses over from the dilute to semi-dilute regime. While $\mathrm{C}<0.40 \mathrm{wt} \%$, the droplet breakup time 
decreases when the Na-CMC concentration is increased at fixed $\theta$ and $\sigma$. While in semi-dilute regime, droplet breakup time increases when if $\mathrm{Na}-\mathrm{CMC}$ concentration is increased. This phenomenon is mainly due to the dispersed phase viscous forces dominating over the breakup dynamics and relevant hydrodynamics. As $\mathrm{Na}-\mathrm{CMC}$ concentration increases, a laminar elongated dispersed thread is formed connecting to the primary droplet due to the high viscous pressure, and thus the droplet breakup point moves progressively downstream of the outlet channel. The presence of high concentration of polymer molecules leads to a prolonged fluid thread and retardation of pinch-off development. In present parametric analysis, there are many features that were previously attributed to elastic effects that still remain a defining challenge for the highly shear-thinning and viscous $\mathrm{Na}-\mathrm{CMC}$ polymer solution. This illustrates the potential of integrating the elastic stress model with present numerical method, in order to investigate the fluid elasticity effect on the growth of droplet with shearthinning characteristics.

\section{Nomenclature}

a Fitting parameter in Carreau-Yasuda model

$d_{\text {eff }} \quad$ Effective droplet diameter $(\mu \mathrm{m})$

$F_{s t}$ Surface tension force acting on the interface $\left(\mathrm{N} / \mathrm{m}^{3}\right)$

$F_{D} \quad$ Cross flow drag force $\left(\mathrm{N} / \mathrm{m}^{3}\right)$

$h \quad$ Depth of the channel $(\mu \mathrm{m})$

I Identity matrix

$k$ Curvature of fluid-fluid interface

$n \quad$ Power-law exponent for Carreau-Yasuda Model

$\mathbf{n}_{\Gamma} \quad$ Unit normal vector at the interface

$p \quad$ Pressure $\left(\mathrm{N} / \mathrm{m}^{2}\right)$

$Q \quad$ Flow rate ratio

$Q_{c} \quad$ Flow rate of the continuous phase $(\mathrm{ml} / \mathrm{hr})$ 
$696 Q_{d} \quad$ Flow rate of the dispersed phase $(\mathrm{ml} / \mathrm{hr})$

$697 \quad t \quad$ Time-step (s)

$698 u \quad$ Velocity component in x-direction $(\mathrm{m} / \mathrm{s})$

$699 \quad v \quad$ Velocity component in y-direction (y-direction) $(\mathrm{m} / \mathrm{s})$

$700 \quad \mathbf{u} \quad$ Velocity field

$701 \quad R \quad$ Curvature radii of the interface $(\mathrm{m})$

702

703 Greek Symbols

$704 \quad \eta \quad$ Dynamic viscosity of fluid (Pa.s)

$705 \quad \eta_{d} \quad$ Dynamic viscosity of the dispersed phase (Pa.s)

$706 \quad \eta_{c} \quad$ Dynamic viscosity of the continuous phase (Pa.s)

$707 \quad \eta_{0} \quad$ Zero shear viscosity (Pa.s)

$708 \quad \eta_{\infty} \quad$ Infinite shear viscosity (Pa.s)

$709 \quad \lambda_{\eta} \quad$ Viscosity ratio $\left(\eta_{d} / \eta_{c}\right)$

$710 \lambda_{C Y}$ Relaxation Time in Carreau-Yasuda Model(s)

$711 \rho \quad$ Fluid density $\left(\mathrm{kg} / \mathrm{m}^{3}\right)$

$712 \quad T \quad$ Shear stress $(\mathrm{Pa})$

$713 \dot{\gamma} \quad$ Shear rate $(1 / \mathrm{s})$

$714 \gamma \quad$ Reinitialization parameter $(\mathrm{m} / \mathrm{s})$

$715 \varepsilon \quad$ Thickness of the interface $(\mathrm{m})$

$716 \sigma \quad$ Interfacial tension $(\mathrm{mN} / \mathrm{m})$

$717 \phi \quad$ Level set function

$718 \delta_{s m}$ Dirac delta function concentrated at interface 
$\Omega \quad$ Computational domain

$\partial \Omega \quad$ Domain boundary

\section{Acknowledgement}

This research project was financially supported by Malaysia Intercampus Doctoral Award Scheme (MIDAS).

\section{References}

Anna, S. L., \& Mayer, H. C. (2006). Microscale tipstreaming in a microfluidic flow focusing device. Physics of Fluids, 18(12), 121512. doi:10.1063/1.2397023

Arratia, P. E., Cramer, L. A., Gollub, J. P., \& Durian, D. J. (2009). The effects of polymer molecular weight on filament thinning and drop breakup in microchannels. New Journal of Physics, 11(11), 115006.

Bashir, S., Rees, J.M., Zimmerman, W.B. (2011). Simulations of microfluidics droplet formation using the two-phase level set method. Chemical Engineering Sciences, 66, 4733-4741.

Bonometti, T., \& Magnaudet, J. (2007). An interface-capturing method for incompressible two-phase flows. Validation and application to bubble dynamics. International Journal of Multiphase Flow, 33(2), 109-133. doi:https://doi.org/10.1016/j.ijmultiphaseflow.2006.07.003

Chhabra, R. P., \& Richardson, J. F. (2008). Chapter 1 - Non-Newtonian Fluid Behaviour. In R. P. Chhabra \& J. F. Richardson (Eds.), Non-Newtonian Flow and Applied Rheology (Second Edition) (pp. 1-55). Oxford: ButterworthHeinemann.

Cramer, C., Fischer, P., \& Windhab, E. J. (2004). Drop formation in a co-flowing ambient fluid. Chemical Engineering Science, 59(15), 3045-3058. doi:https://doi.org/10.1016/i.ces.2004.04.006

Davidson, M. R., \& Cooper-White, J. J. (2006). Pendant drop formation of shearthinning and yield stress fluids. Applied Mathematical Modelling, 30(11), 13921405. doi:https://doi.org/10.1016/j.apm.2006.03.016

Davidson, M. R., Cooper-White, J. J., \& Tirtaatmadja, V. (2004). Shear-thinning drop formation. ANZIAM Journal; Vol 45 (2003).

Deshpande, K. B., \& Zimmerman, W. B. (2006). Simulation of interfacial mass transfer by droplet dynamics using the level set method. Chemical Engineering Science, 61(19), 6486-6498. doi:https://doi.org/10.1016/i.ces.2006.06.012

Garstecki, P., Fuerstman, M. J., Stone, H. A., \& Whitesides, G. M. (2006). Formation of droplets and bubbles in a microfluidic T-junction-scaling and mechanism of break-up. Lab on a Chip, 6(3), 437-446. doi:10.1039/B510841A

Hong, J. S., \& Cooper-White, J. (2009). Drop formation of Carbopol dispersions displaying yield stress, shear thinning and elastic properties in a flow-focusing microfluidic channel. Korea-Australia Rheology Journal, 21(4), 269-280.

Hou, T. Y., Lowengrub, J. S., \& Shelley, M. J. (2001). Boundary Integral Methods for Multicomponent Fluids and Multiphase Materials. Journal of Computational Physics, 169(2), 302-362. doi:https://doi.org/10.1006/jcph.2000.6626 
Huebner, A., Sharma, S., Srisa-Art, M., Hollfelder, F., Edel, J. B., \& deMello, A. J. (2008). Microdroplets: A sea of applications? Lab on a Chip, 8(8), 1244-1254. doi:10.1039/B806405A

Husny, J., \& Cooper-White, J. J. (2006). The effect of elasticity on drop creation in Tshaped microchannels. Journal of Non-Newtonian Fluid Mechanics, 137(1), 121-136. doi:https://doi.org/10.1016/j.jnnfm.2006.03.007

Kobayashi, I., Nakajima, M., \& Mukataka, S. (2003). Preparation characteristics of oil-in-water emulsions using differently charged surfactants in straight-through microchannel emulsification. Colloids and Surfaces A: Physicochemical and Engineering Aspects, 229(1),

33-41. doi:https://doi.org/10.1016/j.colsurfa.2003.08.005

Leshansky, A. M., \& Pismen, L. M. (2009). Breakup of drops in a microfluidic T junction. Physics of Fluids, 21(2), 023303. doi:10.1063/1.3078515

Moon, S.-K., Cheong, I. W., \& Choi, S.-W. (2014). Effect of flow rates of the continuous phase on droplet size in dripping and jetting regimes in a simple fluidic device for coaxial flow. Colloids and Surfaces A: Physicochemical and Engineering Aspects, 454 ,

84-88. doi:https://doi.org/10.1016/j.colsurfa.2014.04.006

$\mathrm{Niu}, \mathrm{X} .$, \& deMello, Andrew J. (2012). Building droplet-based microfluidic systems for biological analysis. Biochemical Society Transactions, 4O(4), 615.

Olsson, E., \& Kreiss, G. (2005). A conservative level set method for two phase flow. Journal of Computational Physics, 210(1), 225-246. doi:https://doi.org/10.1016/i.jcp.2005.04.007

Olsson, E., Kreiss, G., \& Zahedi, S. (2007). A conservative level set method for two phase flow II. Journal of Computational Physics, 225(1), 785-807. doi:https://doi.org/10.1016/j.jcp.2006.12.027

Osher, S., \& Sethian, J. A. (1988). Fronts propagating with curvature-dependent speed: Algorithms based on Hamilton-Jacobi formulations. Journal of Computational Physics, 79(1), 12-49. doi:https://doi.org/10.1016/00219991(88)90002-2

Peng, L., Yang, M., Guo, S.-S., Liu, W., \& Zhao, X.-z. (2011). The effect of interfacial tension on droplet formation in flow-focusing microfluidic device. Biomed Microdevices, 13(3), 559-564. doi:10.1007/s10544-011-9526-6

Qiu, D., Silva, L., Tonkovich, A. L., \& Arora, R. (2010). Micro-droplet formation in nonNewtonian fluid in a microchannel. Microfluidics and Nanofluidics, 8(4), 531548. doi:10.1007/s10404-009-0487-5

Rider, W. J., \& Kothe, D. B. (1998). Reconstructing Volume Tracking. Journal of Computational Physics, 141(2), 112-152. doi:https://doi.org/10.1006/jcph.1998.5906

Shui, L., van den Berg, A., \& Eijkel, J. C. T. (2009). Interfacial tension controlled W/O and OM 2-phase flows in microchannel. Lab on a Chip, 9(6), 795-801. doi:10.1039/B813724B

Steinhaus, B., Shen, A. Q., \& Sureshkumar, R. (2007). Dynamics of viscoelastic fluid filaments in microfluidic devices. Physics of Fluids, 19(7), 073103. doi: $10.1063 / 1.2747660$

Takada, N., Misawa, M., Tomiyama, A., \& Fujiwara, S. (2000). Numerical simulation of two- and three-dimensional two-phase fluid motion by lattice Boltzmann method. Computer Physics Communications, 129(1), 233-246. doi:https://doi.org/10.1016/S0010-4655(00)00110-7 
Tawfik, D. S., \& Griffiths, A. D. (1998). Man-made cell-like compartments for molecular evolution. Nature Biotechnology, 16, 652. doi:10.1038/nbt0798-652

Theberge Ashleigh, B., Courtois, F., Schaerli, Y., Fischlechner, M., Abell, C., Hollfelder, F., \& Huck Wilhelm , T. S. (2010). Microdroplets in Microfluidics: An Evolving Platform for Discoveries in Chemistry and Biology. Angewandte Chemie International Edition, 49(34), 5846-5868. doi:10.1002/anie.200906653

Thorsen, T., Roberts, R. W., Arnold, F. H., \& Quake, S. R. (2001). Dynamic Pattern Formation in a Vesicle-Generating Microfluidic Device. Physical Review Letters, 86(18), 4163-4166. doi:10.1103/PhysRevLett.86.4163

Tirtaatmadja, V., McKinley, G. H., \& Cooper-White, J. J. (2006). Drop formation and breakup of low viscosity elastic fluids: Effects of molecular weight and concentration. Physics of Fluids, 18(4), 043101. doi:10.1063/1.2190469

Tryggvason, G., Bunner, B., Esmaeeli, A., Juric, D., Al-Rawahi, N., Tauber, W., . . . Jan, Y. J. (2001). A Front-Tracking Method for the Computations of Multiphase Flow. Journal of Computational Physics, 169(2), 708-759. doi:https://doi.org/10.1006/jcph.2001.6726

Umbanhowar, P. B., Prasad, V., \& Weitz, D. A. (2000). Monodisperse Emulsion Generation via Drop Break Off in a Coflowing Stream. Langmuir, 16(2), 347351. doi:10.1021/la990101e

Utada, A. S., Fernandez-Nieves, A., Stone, H. A., \& Weitz, D. A. (2007). Dripping to Jetting Transitions in Coflowing Liquid Streams. Physical Review Letters, 99(9), 094502. doi:10.1103/PhysRevLett.99.094502

van der Zwan, E., Schroën, K., \& Boom, R. (2009). A Geometric Model for the Dynamics of Microchannel Emulsification. Langmuir, 25(13), 7320-7327. doi:10.1021/la900379n

Wong, V.-L., Loizou, K., Lau, P.-L., Graham, R. S., \& Hewakandamby, B. N. (2017). Numerical studies of shear-thinning droplet formation in a microfluidic Tjunction using two-phase level-SET method. Chemical Engineering Science, 174, 157-173. doi:https://doi.org/10.1016/j.ces.2017.08.027

Xu, J. H., Li, S. W., Tan, J., \& Luo, G. S. (2008). Correlations of droplet formation in T-junction microfluidic devices: from squeezing to dripping. Microfluidics and Nanofluidics, 5(6), 711-717. doi:10.1007/s10404-008-0306-4

Yobas, L., Martens, S., Ong, W.-L., \& Ranganathan, N. (2006). High-performance flow-focusing geometry for spontaneous generation of monodispersed droplets. Lab on a Chip, 6(8), 1073-1079. doi:10.1039/B602240E

Zhang, X., \& Basaran, O. A. (1995). An experimental study of dynamics of drop formation. Physics of Fluids, 7(6), 1184-1203. doi:10.1063/1.868577 